\title{
Supercritical shallow granular flow through a contraction: experiment, theory and simulation
}

\author{
A. W. VREMAN ${ }^{1,2,3}$, M. AL-TARAZI ${ }^{1}$, J.A. M. KUIPER $\mathbf{S}^{1}$, \\ M. VAN SINT ANNALAND ${ }^{1}$ AND O. BOKHOVE ${ }^{2} \dagger$ \\ ${ }^{1}$ Department of Chemical Engineering, University of Twente, 7500 AE Enschede, The Netherlands \\ ${ }^{2}$ Department of Applied Mathematics, University of Twente, P.O. Box 217, 7500 AE Enschede, \\ The Netherlands \\ ${ }^{3}$ Vreman Research, Hengelo, The Netherlands
}

(Received 10 March 2006 and in revised form 29 November 2006)

Supercritical granular flow through a linear contraction on a smooth inclined plane is investigated by means of experiments, theoretical analysis and numerical simulations. The experiments have been performed with three size classes of spherical glass beads, and poppy seeds (non-spherical). Flow states and flow regimes are categorized in the phase space spanned by the supercritical Froude number and the minimum width of the contraction. A theoretical explanation is given for the formation of steady reservoirs in the contraction observed in experiments using glass beads and water. For this purpose, the classical, one-dimensional shallow-water theory is extended to include frictional and porosity effects. The occurrence of the experimentally observed flow states and regimes can be understood by introducing integrals of acceleration. The flow state with a steady reservoir arises because friction forces in the reservoir are much smaller than in other parts of the flow. Three-dimensional discrete-particle simulations quantitatively agree with the measured granular flow data, and the crucial part of the theoretical frictional analysis is clearly confirmed. The simulations of the flow further reveal that porosity and frictional effects interact in a complicated way. Finally, the numerical database is employed to investigate the rheology in a priori tests for several constitutive models of frictional effects.

\section{Introduction}

In contrast to 'classical' fluid dynamics, the governing constitutive equations describing the dynamics of granular materials are not well-known. This paper records several approaches attempted (often simultaneously) to predict and understand granular dynamics. These include: (asymptotic) continuum theories based on kinetic theory of granular particles (e.g. Lun et al. 1984; Gray, Tai \& Noelle 2003); hydraulic theory applied to granular flows (e.g. Savage \& Hutter 1989; Gray et al. 2003; Hakonardottir \& Hogg 2005); and discrete particle mechanics (e.g. Campbell \& Brennen 1985; van der Hoef et al. 2006; Silbert, Landry \& Crest 2003). The influence of the ambient fluid, such as air, can sometimes be ignored. In other cases the ambient fluid carries the granular material. Examples of carrier fluids are: air in risers; molten metal in the dense conveying of slurries in the metallurgical industry; water in ice flows on rivers in civil engineering; and rivers carrying volcanic debris such as pumice and

$\dagger$ Author to whom correspondence should be addressed: o.bokhove@math.utwente.nl 
tephra in geology. We were originally motivated by a geological case that occurred in the late Pleistocene era (12.900 aBP), in which the Rhine River functioned as the carrier fluid of floating and submerged granular material, or tephra, from the explosion of the Laacher See Volcano. This is estimated to have led to an initially 1-8 $\mathrm{m}$ thick layer of tephra around the volcano (Schmincke 2000). There is evidence that this tephra layer caused dam formation in the Rhine River near the mouth of the Brohltal canyon in the Rhine valley and at a nozzle near Andernach. A large lake then formed extending $50 \mathrm{~km}$ to the southeast (Schmincke 2000), and subsequently the tephra dams collapsed. Can we make a corresponding laboratory experiment and theory in support of this event? The added complexity of a carrier fluid led us to consider the following and experimentally simpler question first: what flow regimes emerge when dry gravitydriven granular matter flows down a smooth inclined chute with a contraction?

Before proceeding with flows through a contraction, a short overview of the literature on granular flows on inclined planes without a contraction is given, in which a distinction is made between granular flows over smooth planes and over rough surfaces. Literature concerning flows on smooth frictional planes includes Augenstein \& Hogg (1978), Brennen, Sieck \& Palaski (1983); Campbell, Brennen \& Sabersky (1985); Johnson, Nott \& Jackson (1990); and Louge \& Keast (2001). Literature concerning flows on rough surfaces often involve a plane with particles glued to the surface (see, for example, Pouliquen 1999; Pouliquen \& Forterre 2002; and GDR MiDi 2004). It is of interest to mention that GDR Midi (2004) contains a phenomenological constitutive law for inclined plane flows over uniform but rough chutes. Obviously, the particles at the bottom experience less slip on rough planes, and their velocity at the wall is relatively small or even zero (no slip). The work of Savage \& Hutter (e.g. Savage \& Hutter 1991) applies to planes of varying roughness. The chute surface in their experiments consists of PVC, writing paper or sandpaper. These surfaces are still relatively smooth; in all cases the bottom roughness is much smaller than the typical size of the flowing grains.

In the rheology of granular flows, Coulomb's law is a basic concept in which tangential stress is simply a constant fraction of the stress normal to the wall. The stress is then entirely frictional and applies to sliding contacts at the bottom. Another important concept is the classic rheological description by Bagnold (1954), who linked the tangential stress to the square of the rate of shear. His experiments and kinetic theory (as e.g. reviewed by Lun et al. 1984) have been very useful in formulating expressions for so-called collisional stresses. In constitutive equations for granular flow, these two concepts are usually combined and the result is a stress defined as a sum of frictional and collisional terms.

Variations in uniform granular flow pertaining to flow around obstacles and oblique granular jumps or 'shock waves' at slight corners have been studied in Gray et al. (2003) and Hakonardottir \& Hogg (2005). In the present paper we consider another variation, granular flow down a smooth inclined plane with contracting sidewalls. Using water instead of granular material, Akers (2005) and Akers \& Bokhove (2007) performed and analysed experiments on a flow on a horizontal plane, constrained downstream by contracting sidewalls. Key parameters to classify the hydraulic, as well as the granular, flow regimes are the upstream Froude number $F_{0}$ and the scaled nozzle width at the end of the contraction $b_{c} / b_{0}$, with $b_{0}$ the constant width of the channel upstream of the contraction. The Froude number $F_{0}=u_{0} / \sqrt{g_{n} h_{0}}$ is the ratio of the average upstream velocity $u_{0}$ down the chute and the surface gravitywave speed $\sqrt{g_{n} h_{0}}$ with $h_{0}$ the mean constant depth and $g_{n}$ the component of the acceleration due to gravity normal to the plane. 

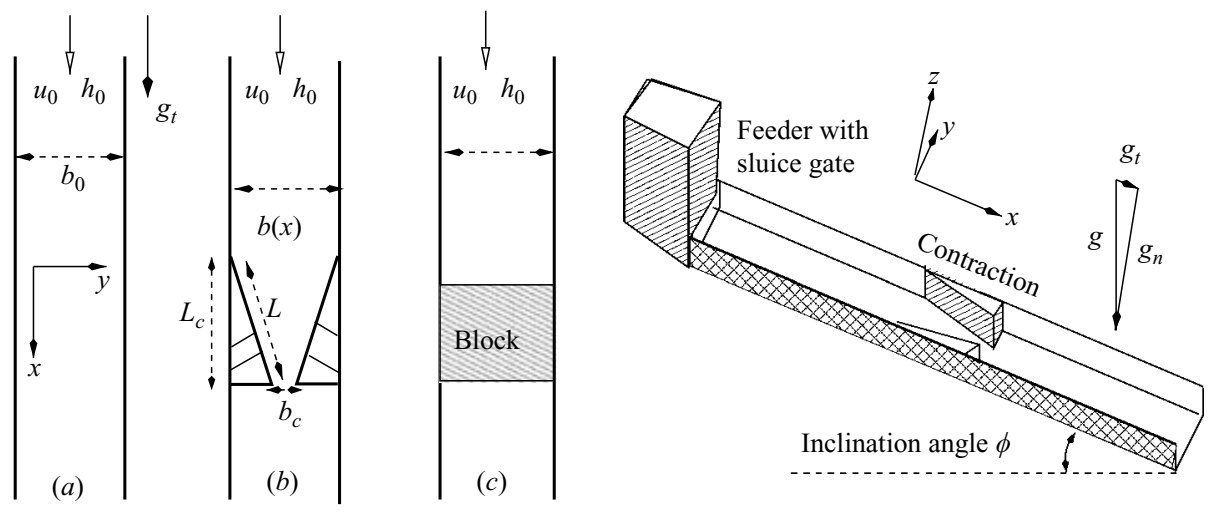

FIGURE 1. Top and side view sketches of the inclined chute experiments: $(a)$ of constant width, $b(x)=b_{0} ;(b)$ with a localized contraction; and $(c)$ blocked in the middle, ' $b_{c}=0$ '.

The paper's objectives and outline are as follows. First, we classify the flow regimes and states in laboratory experiments on granular flow down an inclined chute with a contraction $(\$ 2)$. The chute has uniform width $b_{0}$ except for a linear contraction placed in the middle. To limit our study the following constraint is imposed: if we remove the contraction then the flow is not subject to visible surface or density waves and is as uniform as possible for a given gap height $h_{l}$ at the upstream sluice gate. This constraint determines the inclination angle $\phi$ of the chute and leads to an approximate balance between the downstream force of gravity on the granular particles and the average inter-particle and particle-wall forces. In this way, the Froude number is largely fixed. To obtain a larger range of Froude numbers $F_{0}$ we varied the type of granular material, as well as the gap height at the sluice gate. We used three sizes of spherical glass beads with small, mean and large diameters, and non-spherical particles (poppy seeds).

Second we present an extended or novel granular 'hydraulic' theory explaining the observed flow regimes and states, based on an analysis of one-dimensional equations $(\S 3)$. It is an extension of classical inviscid hydraulic theory because effects of friction and compressibility are accounted for. The use of acceleration integrals in our approach is novel, as it does not require a closure based on constitutive equations to relate theory and experimental data.

Third we explore the observed and analysed granular flow states in detail and, in particular, confirm our theoretical explanation of the reservoir state by analysing discrete-particle simulations $(\S 4)$. Computer power is here the limiting factor as a large number of particles is required to represent the flow realistically.

Finally, we analyse several theories for friction in granular flows, comparing them in detail with friction and granular temperature data available from the numerical database of the discrete-particle simulations (§5). The rheological implications of this analysis give further insight into the frictional behaviour of granular flow inside a contraction.

\section{Experiments}

In the following subsections, we will describe the experimental set-up and the experiments performed, and conclude by classifying the results in a phase diagram. The experiments and the three flow states are illustrated in figures 1 and 2. In 

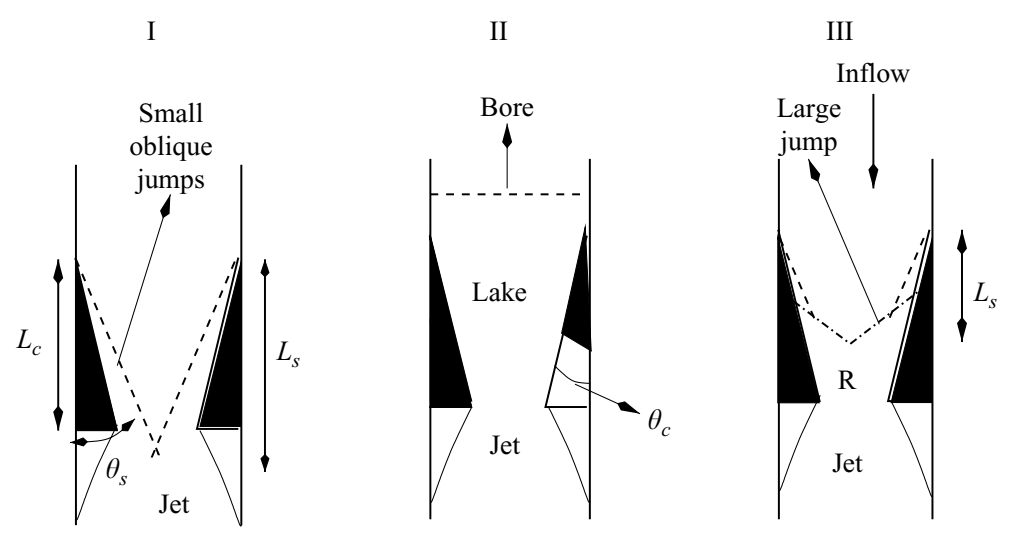

FIGURE 2. A classification sketch of the three flow states include, in a top view of the chute: (I) steady supercritical flow for $b_{c} \lesssim b_{0}$; (II) flow with a lake and an upstream travelling or steadied bore for $b_{c} \gtrsim 0$; and, (III) steady flow with a reservoir, denoted by "R". All cases have a jet behind the contraction. Dashed(-dotted) lines are jump or bore fronts.

our experiments we observed three possible states for supercritical flows $\left(F_{0}>1\right)$ by varying the Froude number and the contraction width $b_{c}$. The crucial question is when and why the transitions between these states occur.

\subsection{Description of experiments}

Initially the granular material is in a storage tank or feeder, which is fitted with a funnel down to the top stretch of the chute behind the sluice gate. A gap height of the sluice gate is set and can be varied between 0 and $13 \mathrm{~mm}$. The inclination angle $\phi$ of the chute is adjustable to between 0 and $35^{\circ}$. The bottom and sidewalls of the chute are made of aluminum. The length of the chute is $2 \mathrm{~m}$ and its width $b_{0}=0.13 \mathrm{~m}$. The experimental set-up is sketched in figure 1. An experiment is started by opening the valve in the funnel of the storage tank. The granular material piles up against the sluice gate and then gradually flows down the slope of the chute. At the end, the granular material is collected in a bin placed on an electronic balance connected to LabView software. The mass flux as function of time as well as the steady state can thus be determined, with an average mass discharge within $1 \%$ error. Metallic rods and meshes are placed in the feeder and collecting bin. These, together with the chute itself, are earthed to minimize electrostatic effects. The velocity of the granular particles at the top of the granular layer is measured using particle image velocimetry (PIV) in a selected section of the chute of about $0.20 \mathrm{~m}$ in length.

The linear contraction is formed by a pair of triangular-shaped aluminum wedges (see figure $1 b$ ). For most experiments a pair with hypothenuse of $L=0.201 \mathrm{~m}$ was used, but a few experiments were performed with a pair of wedges with hypothenuse of $0.40 \mathrm{~m}$. The line connecting the sharp corners of the wedges is the contraction entrance, which is set at $0.60 \mathrm{~m}$ from the sluice gate, unless otherwise noted. The narrowest width $b_{c}$ of the contraction could be varied continuously between 0 and about $0.10 \mathrm{~m}$ by rotating the wedge around its sharpest corner touching the chute wall. From the hypothenuse and the gap width $b_{c}$, one can calculate the length of the wedge along the chute with Pythagoras' rule.

We performed nine series of experiments for dry granular material. Eight series (S18) were performed for three different almost spherical glass beads of multi-disperse size: (S) small diameter $d \in[0.28,0.42] \mathrm{mm}$, (M) medium $d \in[0.4,0.6] \mathrm{mm}$, and (L) 
large $d \in[0.75,1.0] \mathrm{mm}$. In these series the beads were not sieved by us, but used in the size class and type as delivered by Sigmund Lindner. $\dagger$ The material density of the glass beads was $\rho_{p}=2470 \mathrm{~kg} \mathrm{~m}^{-3}$. We performed one series of experiments with poppy seeds $\uparrow$ Unlike spherical glass beads, a typical poppy seed has a banana-like shape with an approximate length of $1.0 \mathrm{~mm}$, and an approximate diameter of $0.7 \mathrm{~mm}$ in the middle. The density $\alpha_{\max } \rho_{p}$ in static random packing equals $616 \mathrm{~kg} \mathrm{~m}^{-3}$ with $\alpha_{\max }$ the solid volume fraction. In addition, we performed two series of experiments with water. It was instructive to observe the behaviour of an incompressible fluid (water) in the same experimental set-up. To keep the focus on granular flows, a discussion of these experimental results is relegated to Appendix A.

To measure the depth of the granular layer we used a ruler with electronic display, applied perpendicularly to the chute's surface relative to the level sidewalls of the chute. The ruler was carefully moved down until its tip hit the surface of the flow, with some granular particles bouncing against the tip, the ruler was yet sufficiently high to avoid a visible wake in the granular layer. The error in these depth measurements of the uniform flows is about $0.1 \mathrm{~mm}$, which corresponds to a maximum relative error of $6 \%$.

For each set of experiments, the measurements were performed on the same day. Each measurement of granular flow through the contraction is preceded by an experiment without a contraction to establish nearly uniform flow conditions sufficiently far from the transients near the sluice gate. A flow is adjusted to be approximately uniform by changing the inclination angle $\phi$ such that variations in depth and velocity of the particles at the free surface along the chute are minimal. Depth, velocity and porosity measurements of the flow were taken around $0.60 \mathrm{~m}$ downstream of the sluice gate. In most cases we started with a relatively low inclination for which the material did not flow regularly. Then we gradually increased the inclination until the depth could be measured adequately, since surface waves ceased to bounce against the ruler's tip. At that point we analysed the top-layer velocity field obtained by PIV $0.60 \mathrm{~m}$ from the sluice gate to validate that the variation of the streamwise velocity in the streamwise direction was small. From the PIV measurements we deduced that the flow at this chosen inclination was uniform or slightly accelerating: that is, the measured PIV-velocity increased less than $10 \%$ within the camera window (of $0.15 \mathrm{~m}$ ). The latter error is an upper bound; typical values were $2 \%, 4 \%$ and $6 \%$. For a typical streamwise velocity of $0.5 \mathrm{~m} \mathrm{~s}^{-1}$, this implies an acceleration of at most $0.1 \mathrm{~m} \mathrm{~s}^{-2}$. In two cases with a reservoir (small particles and water), we verified the nearly uniform character of the upstream flow by repeating the experiment with the contraction placed further downstream, and in both cases a similar reservoir state reappeared. The inclination angle was measured with a protractor supplied with a spirit level $\left(0.25^{\circ}\right.$ measurement error $)$. Note that, while performing the procedure described above, we observed a single inclination angle rather than a range of angles for which approximately uniform flow without visible density waves occurred.

The volume fraction of the particles was measured by a trapping method (Pouliquen 1999). A cup without a bottom was suddenly placed on the surface of the chute to trap the mass in the flow in a surface area $A=0.0020 \mathrm{~m}^{2}$. Then the material in the cup was weighted. To estimate the error, 16 repeated measurements were taken for a specific flow of small-size particles, in which we measured an average weight of $2.9 \mathrm{~g}$

$\dagger$ Sigmund Lindner, GmbH, Warmersteinach, Germany, www.sigmund-lindner.com; SiLibeads with Art. Nos. 45015, 4503 (type S) were used.

$\ddagger$ DIPASA Europe in Enschede, The Netherlands, www.dipasa.nl; with thanks to Oscar Woltman. 
with a standard deviation of $0.2 \mathrm{~g}$, giving a measurement error of about $7 \%$. (The balance at the bottom of the chute was used to collect and weigh trapped particles.) With the measured mean layer depth $h_{0}$ and given the material density, the error in particle volume fraction $\alpha$ is then about $13 \%$. The mean velocity $u_{0}$ of the flow along the chute can be expressed in terms of the measured mass flux $Q$, the weight $m_{\text {cup }}$ measured in the cup and the material density $\rho_{p}: u_{0}=Q A /\left(b_{0} m_{\text {cup }}\right)$. Note that $h_{0}$ is not necessary to calculate $u_{0}$. The relative error in $u_{0}$ is about $7 \%$, since the inaccuracy in $u_{0}$ is mainly caused by $m_{\text {cup }}$. The Froude number

$$
F_{0}=u_{0} / \sqrt{g_{n} h_{0}}
$$

of the flow $0.60 \mathrm{~m}$ downstream of the sluice gate is calculated with an error of about $10 \%$. Here $g_{n}=g \cos \phi$ is the normal component of the acceleration vector of gravity of magnitude $g=9.8 \mathrm{~m} \mathrm{~s}^{-2}$.

For granular material, the Froude number $F_{0}$ and the inclination angle $\phi$ determined by the procedure described above depend on the weather conditions, the dryness of the material and the state of polishing by wear and tear of the beads. Despite these changes, measurements (sets S1 to S11) of the flows through the contraction were set against the valid and established nearly 'uniform' flow state on that day. $\dagger$

\subsection{Experimental results}

The experiments concern flow of granular material in a shallow layer with a free surface. Relatively sudden steady or moving jumps in the free surface and the velocity were observed; these are granular jumps and bores, akin to hydraulic jumps and bores in shallow water flows. We consider shallow flows that are supercritical upstream of the contraction such that $F_{0}>1$. The Froude number is the incompressible analogue of the Mach number in compressible flows. Although shallow granular flows are often modelled as incompressible, they can be compressible and we therefore use the words 'jump', 'bore' and 'shock' interchangeably. Thus, shock waves are expected to arise due to a sufficiently narrow contraction, in which the flow slows down, and large jumps appear for relatively large $F_{0}$.

Table 1 summarizes the experimental reference conditions without the contraction measured $60 \mathrm{~cm}$ downstream of the sluice gate. Each set corresponds to a series of contraction experiments with fixed $F_{0}$ and varying $b_{c}$. After a series of experiments, the contraction wedges were removed to verify whether the same reference state was still replicable. $\ddagger$

The following two different main flow states were observed: (i) The flow was relatively smooth during the entire experiment, also in the contraction. (ii) A bore with a large jump was formed near the contraction exit and travelled upstream. Most travelling bores observed stopped before reaching the sluice gate. When the bore

$\dagger$ All measurements were performed in a dry atmosphere (with humidity around $20 \%$ and a temperature around $30^{\circ} \mathrm{C}$ due to the illumination). One series of experiments (S0) was previously documented in an internal report (Al-Tarazi et al. 2006). The results of both experiments are consistent.

$\mp$ In set $\mathrm{S} 0$ a different approach was used to obtain $F_{0}$ since the volume fraction was not measured at the entrance of the contraction, but $0.20 \mathrm{~m}$ before it. Therefore, $u_{0}$ was derived from the PIV-velocity $\left(0.48 \mathrm{~m} \mathrm{~s}^{-1}\right.$ according to figure 4$)$ while assuming that the ratio between $u_{0}$ and PIV top-velocity equalled the corresponding measured ratio for set S5, in other words the set with similar particle diameter, at $85 \%$. The value of $h_{0}$ was taken by averaging the eight values measured around the entrance of the contraction (which can be found in figure 8). Finally, the value of $\alpha_{0}$ for series S0 was calculated from the mass flux $Q, u_{0}$ and $h_{0}$. 


\begin{tabular}{rlcccccc}
\hline Set & \multicolumn{1}{c}{ Fluid } & $F_{0}$ & $u_{0}\left(\mathrm{~m} \mathrm{~s}^{-1}\right)$ & $h_{0}(\mathrm{~mm})$ & $\alpha_{0}$ & Sluice gate $(\mathrm{mm})$ & $\phi$ (deg.) \\
S0 & 0.55 mm beads & 2.9 & 0.41 & 2.15 & 0.35 & 4 & 15.5 \\
S1 & small beads & 3.9 & 0.48 & 1.7 & 0.34 & 4 & 24 \\
S2 & small beads & 4.7 & 0.94 & 4.5 & 0.43 & 8 & 24 \\
S3 & small beads & 5.5 & 0.67 & 1.7 & 0.29 & 4 & 26 \\
S4 & small beads & 3.5 & 0.44 & 1.8 & 0.35 & 4 & \\
S5 & medium beads & 3.0 & 0.44 & 2.35 & 0.34 & 4 & 19.5 \\
S6 & large beads & 2.0 & 0.33 & 3.0 & 0.25 & 4 & 19 \\
S7 & large beads & 2.6 & 0.54 & 4.7 & 0.32 & 8 & 19 \\
S8 & large beads & 2.8 & 0.58 & 4.7 & 0.32 & 8 & 3 \\
S9 & water & 3.7 & 0.48 & 1.7 & 1 & 4 & 3 \\
S10 & water & 4.0 & 0.50 & 1.6 & 1 & 4 & 20 \\
S11 & poppy seeds & 5.2 & 1.0 & 3.9 & $0.64 \alpha_{\max }$ & 13 &
\end{tabular}

TABLE 1. The reference flow values in the absence of a contraction for the twelve sets of experiments. Set S0 comes from an earlier experiment with glass beads of diameter $d=0.55 \pm 0.05 \mathrm{~mm}$ (Al-Tarazi et al. 2006). The error in $\alpha_{0}$ is $13 \%$.

(a)

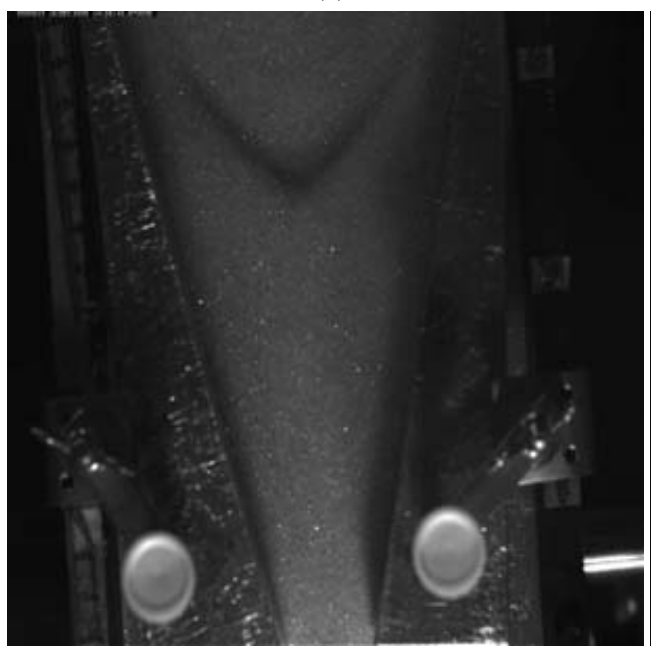

(b)

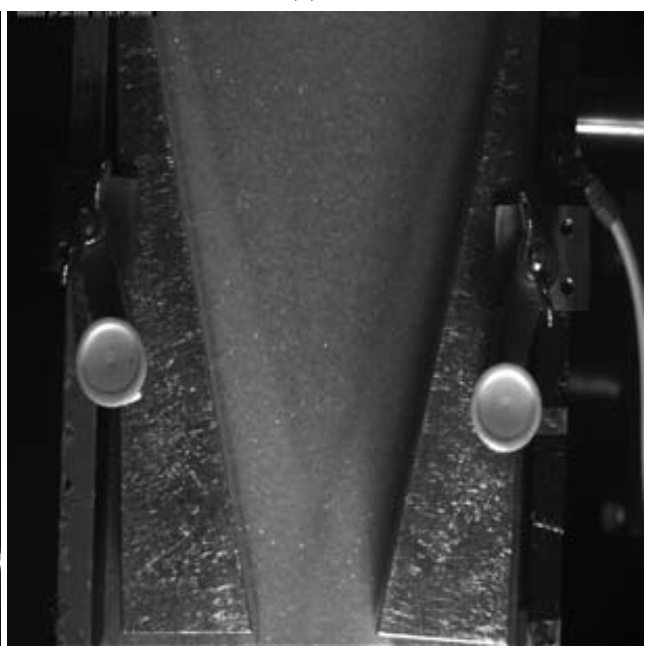

FIGURE 3. Snapshots from experiments: $(a)$ a steady granular reservoir for the smallest size class of glass beads, and $(b)$ smooth granular flow with two oblique shocks involving small particles.

remained steady for more than $10 \mathrm{~s}$, we concluded that a steady lake had formed. The flow between the bore front and the exit of the contraction is called a lake when the granular jump is outside the contraction, and is called a reservoir when it is inside. In a lake or reservoir the depth is considerably larger than in other parts of the flow. However, for very small $b_{c}$ the bore front continued to move backward, until the feeder was empty (the duration of an experiment was typically about one minute).

Snapshots of experiments are shown in figures 3 and 4 for smooth flow with weak oblique shocks, and steady reservoirs for different sizes glass beads. In figure $3(a)$, the front of the shock as the starting point of the reservoir is clearly recognizable. The front is V-shaped for the granular flow. When $b_{c}$ is decreased the shock halts further upstream and the reservoir runs into a lake upstream of the contraction. Depth measurements are shown in figure 5 for a steady granular reservoir and a water 


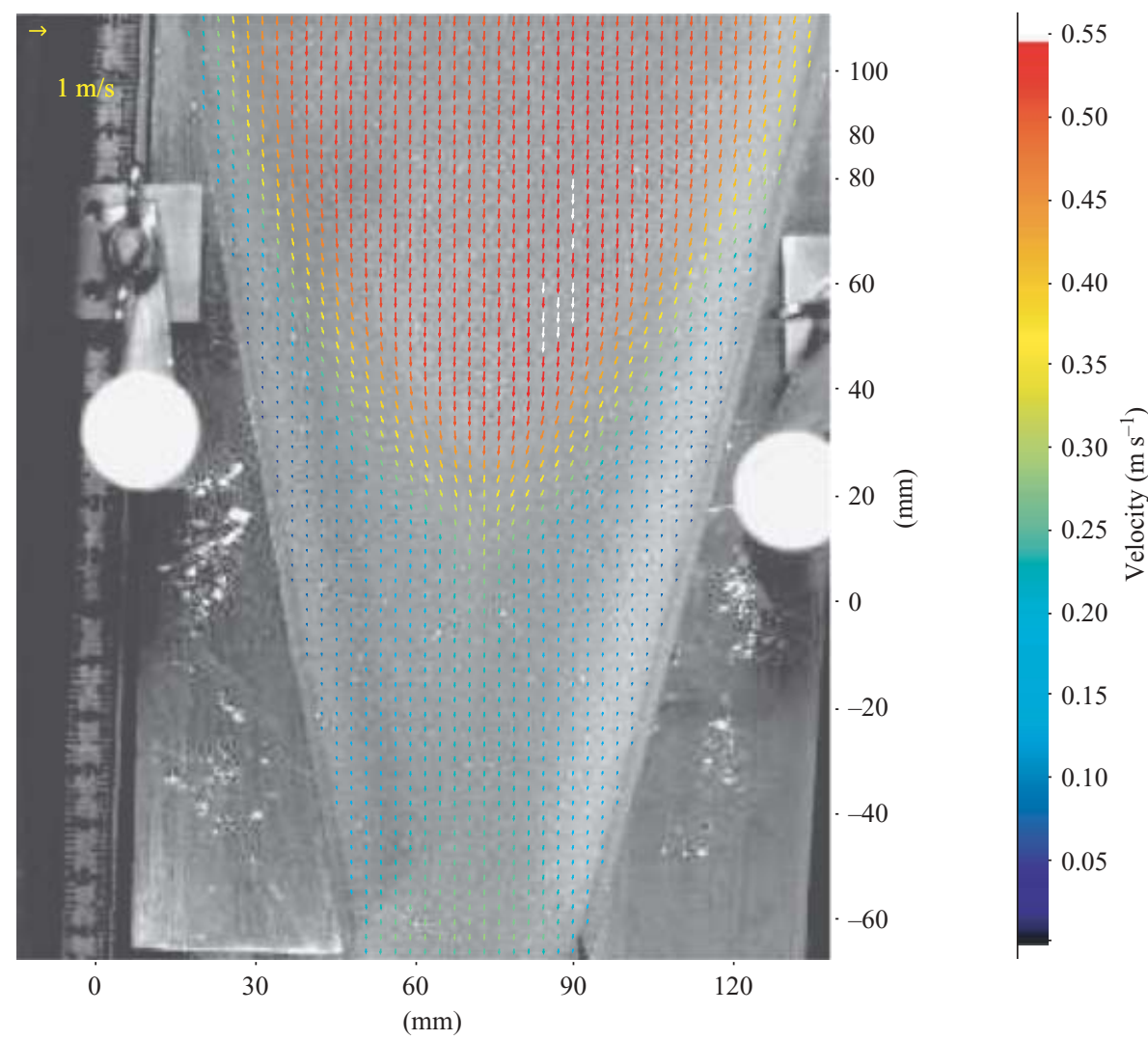

FIGURE 4. A snapshot of the granular flow with a steady reservoir for $b_{c}=44 \mathrm{~mm}$ in experiment S0, see table 1 .

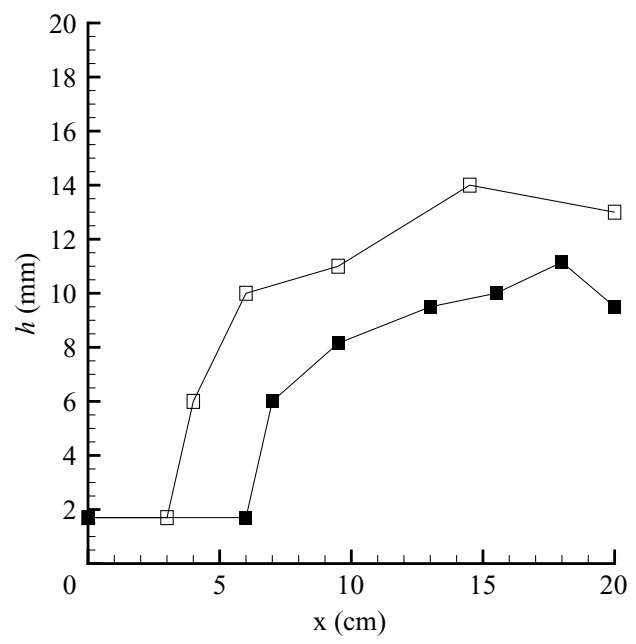

Figure 5. The depth $h=h(x, y)$ of the shallow layer is shown along the centreline of the chute as a function of the streamwise coordinate $x$. The contraction starts at $x=0$ and ends at $x=0.20 \mathrm{~m}$. Shown are experiments $\mathrm{S} 1$ with $b_{c}=26 \mathrm{~mm}$ for small particles and $F_{0}=3.9$ (solid symbols) and S10 with $b_{c}=21 \mathrm{~mm}$ for water and $F=4.0$ (open symbols). 
reservoir. In both cases, the depth suddenly increases across the shock by a factor 3 to 5 , whereafter it increases more slowly to attain a maximum several centimetres before the nozzle exit.

When $b_{c}$ is increased sufficiently, relatively smooth flow occurs with weak oblique shocks created by the sudden change of the angle of the sidewall relative to the mean flow direction. These weak oblique shocks are observed in figure $3(b)$ and are similar to the those observed by Gray et al. (2003), and also in Hakonardottir \& Hogg (2005) where a single wedge is placed in a uniform flow. For a sufficiently large value of $b_{c}$ the oblique shocks do not cross before the nozzle exit. The typical jump in the depth $h$, about a factor of 2 for these oblique shocks, is smaller than for the reservoir front. After averaging in the transversal direction, the averaged depth $h$ smoothly increases from the start of the contraction to at least the point where the shocks cross - if they cross. The steady reservoir cases are clearly distinguishable from these 'smooth' flows. When a reservoir is created, it has been preceded by oblique shocks in the transient stage. From the point where these shocks crossed, near the end of the contraction, a bore developed, travelled backwards, and then covered the oblique shocks until it halted and formed a steady reservoir. The remainders of the oblique shocks are still visible in figures $3(a)$ and 4 . They form the outer edges of the V-shape. After the exit of the contraction a granular jet occurs in all cases, as in figure 6; the flow freely develops here until, about $0.10 \mathrm{~m}$ further downstream, the flow reattaches to the sidewalls.

For experiments S2, S3, S10 and S11 at the three highest Froude numbers for $F_{0} \gtrsim 4.0$, we observed that the flow was able to attain multiple states for appropriate values of $b_{c}$. This phenomenon is called hysteresis (Baines \& Whitehead 2003). In each observed case of hysteresis, a steady smooth flow developed when the flow was started gradually after opening the storage tank. In contrast, a lake with a bore formed when a dam-break scenario started the experiment. When we manually disturbed the former smooth flow by partially blocking the contraction exit for a short time, the smooth flow state changed to the lake state or reservoir state, see figure 6. This latter lake state was stable in the sense that it did not disappear spontaneously. However, when we pushed enough granular material from the lake through the exit, then the original smooth flow state reappeared.

The experimental results are collected in the $F_{0}, b_{c} / b_{0}$ phase diagram in figure 7 for the flows through a contraction. We distinguish three flow states and four flow regimes, denoted by four different symbols. The three states are: (I) smooth supercritical flow, (II) an upstream moving bore or a steady lake halting outside the contraction, (III) a steady reservoir with a strong jump inside the contraction. Regimes (I), (II) and (III) are regions in the phase space, where the corresponding state is unique. Multiple flow states were observed in regime (IV), where state (I) spontaneously occurred (when the experiment started with an empty chute and a fixed contraction width), but changed into (II) or (III) after a sufficiently strong external perturbation. We found these hysteretic flows for $F_{0} \gtrsim 4.0$. For set S10 with $F_{0} \approx 4$ we observed all flow regimes in a single set of experiments.

All measurements reported in this paper are for the wedge length $L=0.201 \mathrm{~m}$; a longer set of wedges was only used in additional experiments, not discussed here. We found that a reservoir in a contraction of $0.201 \mathrm{~m}$ also occurred in the longer contraction with $L=0.40 \mathrm{~m}$ for the same $b_{c}$. In both cases the length of the reservoir was roughly the same. In the same way, a lake that halted several centimetres outside the $0.201 \mathrm{~m}$ contraction (state III) was found to be entirely inside the $0.40 \mathrm{~m}$ contraction, where it would be called a reservoir. Thus, the precise demarcation 
(a)

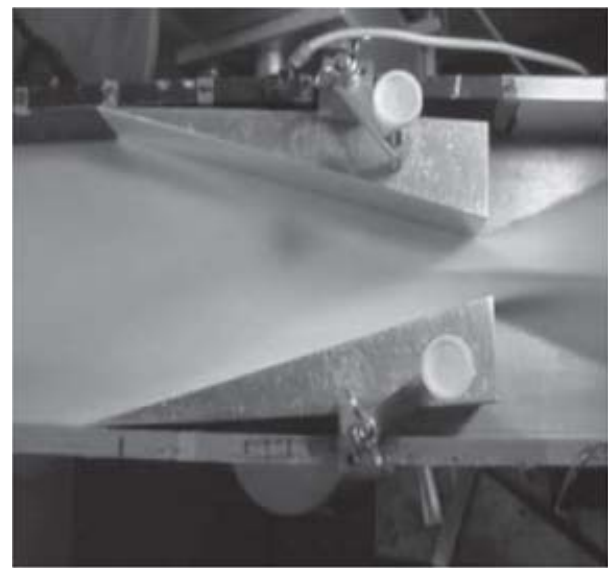

(c)

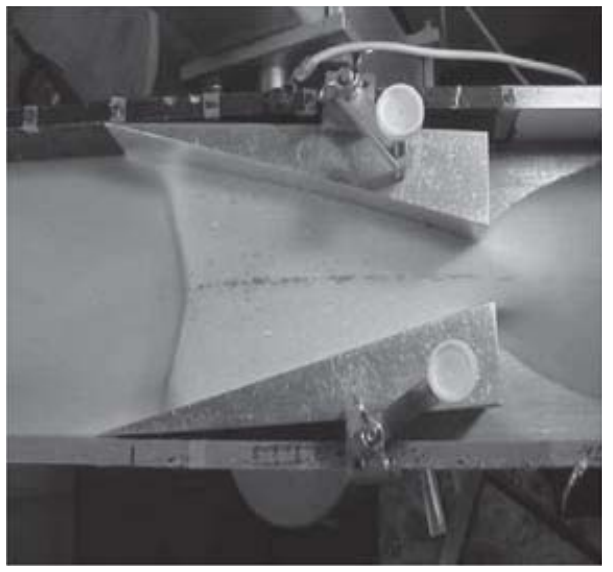

(b)

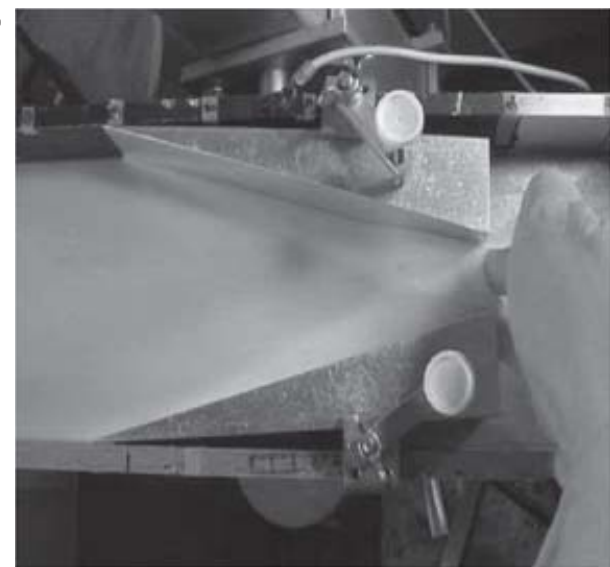

(d)

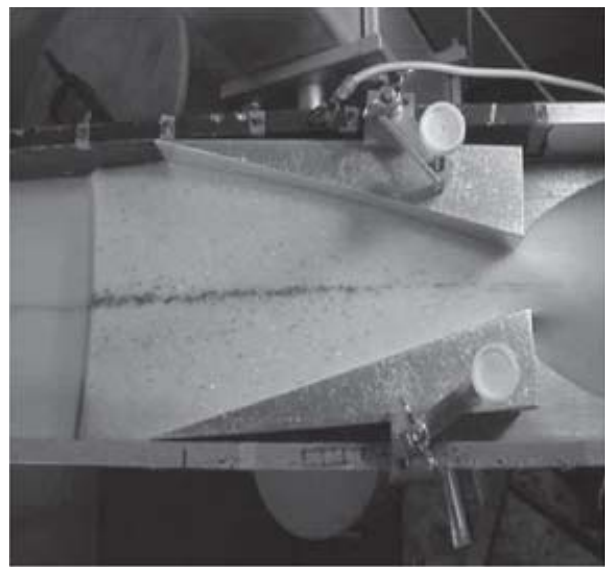

FIGURE 6 . When we manually disturb the smooth flow by partially blocking the contraction exit for a short time, here with a finger, the smooth flow state changes to the lake state. This transition is displayed in $(a)-(d)$. (a) Flow with smooth oblique jumps and $(d)$ flow with an upstream halted jump and lake. All cases clearly show the jet after the contraction exit.

between state II and III depends on $L$. Nevertheless, we do distinguish between a reservoir inside the contraction and a steady lake outside the contraction, because the separating curve (a reservoir that starts at the entrance of the contraction) is suitable for an analytic approach, which provides insight into the physical mechanism that creates a reservoir of a certain length (see $\S 3$ ).

In figure 7, a solid line is drawn to separate regime IV with multiple flow states from the lake regime II for $F_{0} \gtrsim 4.0$, and to separate the smooth flow regime I from the reservoir regime III. Similarly, a dashed line is drawn to separate smooth flow regime I from the multiple flow regime IV for $F_{0} \gtrsim 4.0$, and reservoir state III from the lake regime II. These curves are essential to further our understanding of these experiments, and we will present a theory to predict them in the next section. To the right of the solid curve, the supercritical flow state (co-)exists, whereas to the left of the curve the lake or reservoir states II or III exist with a subcritical region. For the supercritical flow, the Froude number $F=u / \sqrt{g_{n} h}>1$ everywhere including at the nozzle exit where $F_{c}=u_{c} / \sqrt{g_{n} h_{c}}>1$ (the depth and velocity at the exit are denoted as $h_{c}$ and $u_{c}$ ). In contrast, when a subcritical flow state occurs then $F_{c}=1$ at the nozzle exit, because the flow goes through a shock wave upstream of the nozzle exit. 


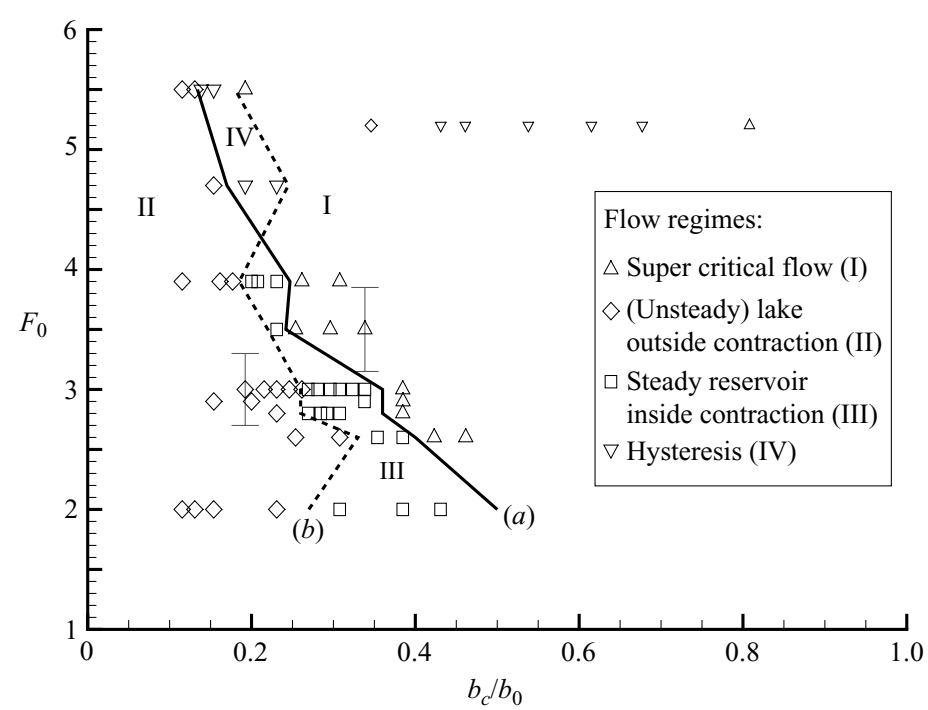

FiguRE 7. Experimental granular results collected in a phase diagram of $F_{0}$ versus $b_{c} / b_{0}$. Smaller symbols denote poppy seed experiments. Flow regimes observed: (I) smooth supercritical flow, (II) a steady lake halting outside the contraction or upstream moving bore, (III) a steady reservoir with a granular or hydraulic jump inside the contraction, and (IV) a hysteretic region with multiple flow states. Solid and dashed lines demarcate transitions between (a) regimes II-IV and III-I, and (b) regimes IV-I and II-III. Two representative error bars are shown.

Across this shock, the upstream supercritical Froude number suddenly drops to a subcritical value $F_{s}<1$, where $F_{s}$ is the Froude number directly downstream of the shock front. Between this location $x=x_{s}<x_{c}$ of the shock and the contraction exit at $x=x_{c}$, the Froude number increases to $F_{c}=1$ and downstream of the contraction the flow is again supercritical.

Classical hydraulic theory (Baines \& Whitehead 2003) or Lavalle-nozzle theory of compressible flows (Shapiro 1953) applied to our flow predicts that the streamwise velocity $u$ either attains a minimum at $x_{c}$ for entirely supercritical flows, or is critical with $F_{c}=1$ at the nozzle exit. The corresponding mathematics will be shown in the next section when we extend this theory. We have experimentally verified the classical theoretical prediction that $F_{c}=1$ at the nozzle exit for flows with a reservoir. For a range of $b_{c}$ in which there is a switch from smooth flows to flows with either a steady reservoir or lake, we measured the depth $h=h_{c}$ of the layer at the contraction exit. We did this for the singlet flow type with small particles (S4) and for the hysteretic flow type with water (S10). When the depth and the porosity are known, $F_{c}$ is known, since we can then calculate the velocity $u_{c}$ from the measured steady mass flux. The values of $h_{c}$ and $F_{c}$ are shown in figure 8, using the same symbols as in figure 7. It is clear that $F_{c} \approx 1$ for the cases with a reservoir or lake (the squares in the figure). Increasing $b_{c}$ produces smooth solutions (the triangles in the figure) and we observe that $F_{c}$ smoothly increases with $b_{c}$. For a given $F_{0}$, the minimum $b_{c}$ for all smooth solutions corresponds to $F_{c} \approx 1$, verifying the validity of hydraulic theory. We conclude therefore that the experimental solid demarcation line in figure 7 corresponds to $F_{c}=1$.

To calculate $F_{c}$ for the granular data in figure 8 , we indirectly measured the volume fraction $\alpha_{c}$. We used a different cup then before (of rectangular shape such that it fitted in the lake) and trapped material just before the end of the contraction. For the 

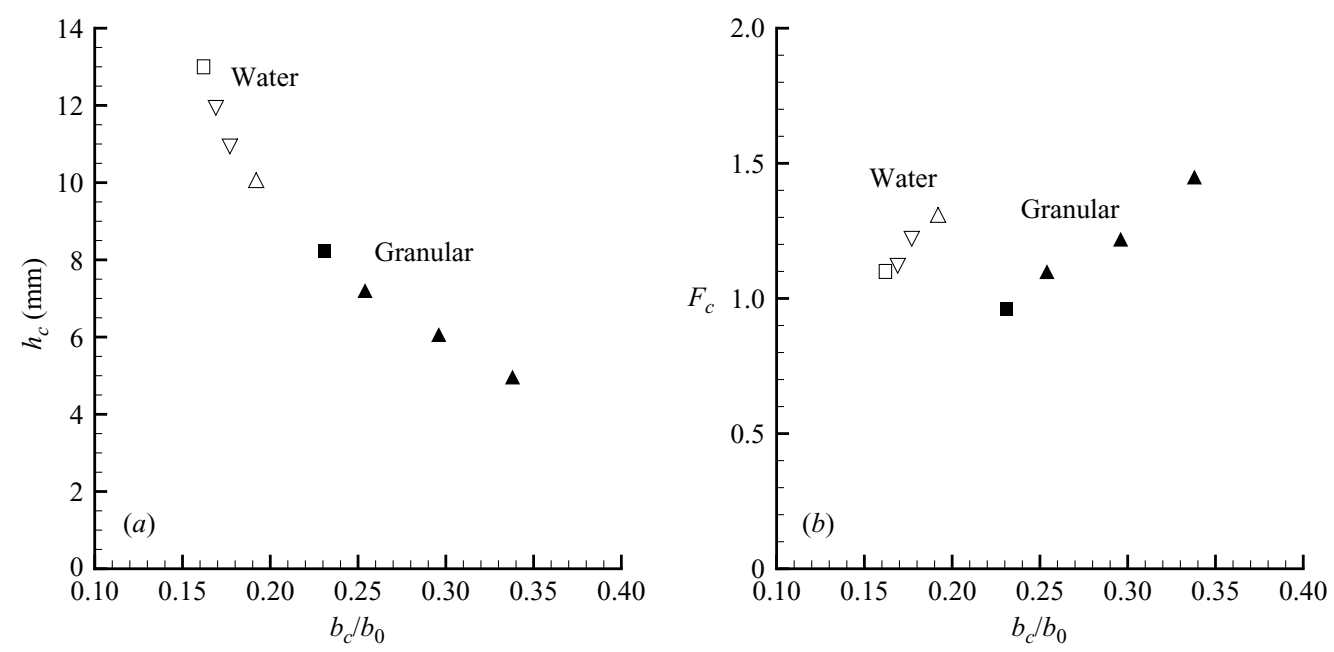

FIGURE 8. (a) Depth and $(b)$ Froude number at the nozzle exit $x_{c}$ for the experimental data sets S4 (solid symbols) and S10 (open symbols). See figure 7 for the meaning of the symbols. The values of $h$ for the hysteretic regime concern the smooth flow state (downward pointing triangles).

lowest value of $b_{c}$ we measured $\alpha_{c}=0.56$, which was also used for the granular $F_{c}$ for the larger $b_{c}$ in figure 8 . In these cases there was no lake and, consequently, the depth $h$ is not constant across the surface of the cup, which makes determination of $\alpha$ unreliable for these smooth flow cases. As the value of $\alpha_{c}$ for smooth flow is likely to be lower than for flow with lake or reservoir flow, the solid triangles for $F_{c}$ in figure 8 should be interpreted as lower bounds for the value of $F_{c}$. We also measured $\alpha$ inside a number of granular reservoirs and lakes and typically found values around 0.6 , very close to the maximum packing value of about 0.64 . Only for lakes in the sets with the large particles (S6-8), we measured a lower value $\alpha \approx 0.52$. Compared to the reference values of the volume fraction tabulated in table 1 , it is clear that the volume fraction significantly increases in a reservoir. The influence of variations in the volume fraction on shock speeds will be discussed later.

For series S0, depth and volume fraction measurements are shown in figure 9, which gives an overview of the steady adjacent states with a lake with a moving bore halted against the sluice gate (circles), a lake with a jump halted due to friction (triangles), a reservoir in the contraction (squares), and smooth flow with oblique shocks (crosses).

Contour plots of the measured streamwise velocity for small particles (S1) and large particles (S8) are shown in figure 10. The two reservoir states demonstrate that the velocity $u$ suddenly reduces across the shock front. By comparing figures $10(a)$ and $(c)$, we see that the strength of this reduction increases with Froude number. Just after the shock front, the velocity increases again, but at the exit the velocity is still lower than the free-stream value. In particular figures $10(b)$ and $10(d)$ demonstrate that the sidewalls influence the velocity also if there is no contraction. The crossand depth-averaged velocity, $u_{0}=0.48 \mathrm{~m} \mathrm{~s}^{-1}$ for $\mathrm{S} 1$ and $0.58 \mathrm{~m} \mathrm{~s}^{-1}$ for $\mathrm{S} 8$, on which the Froude number is based, is considerably lower than the surface velocity at the centre of the chute, 0.55 and $0.7 \mathrm{~m} \mathrm{~s}^{-1}$, respectively. Hence, cross-averaging leads to an average surface velocity of approximately $90 \%$ of the peak velocity measured at the surface by PIV. The combination with depth-averaging then leads to the following 

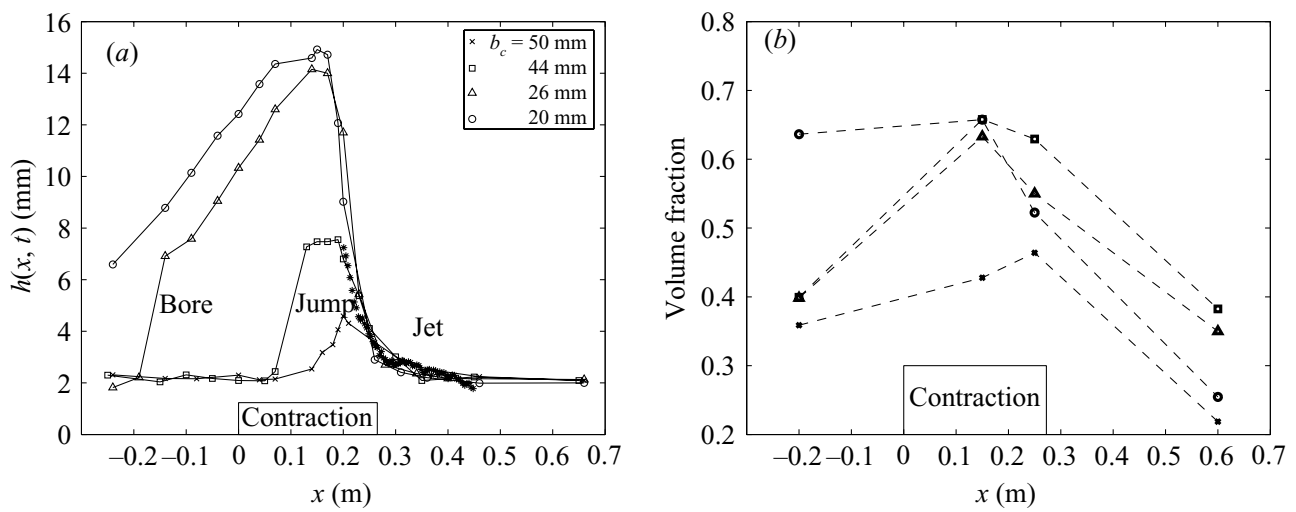

FiguRE 9. (a) The measured layer thickness (solid lines) and $(b)$ the volume fraction (dashed lines) versus $x$ are shown for various $b_{c}$ and with $F_{0}=2.9$ and $\phi=15.5^{\circ}: b_{c}=50 \mathrm{~mm}$, nearly smooth supercritical flow; $b_{c}=44 \mathrm{~mm}$, a reservoir with steady granular jump; and, $b_{c}=20$ and $26 \mathrm{~mm}$, a reservoir with upstream moving granular bore. The stars for case $b_{c}=44 \mathrm{~mm}$ correspond to discrete hard-sphere particle simulations starting at the nozzle. Volume fraction is measured by trapping particles in a cup. From Al-Tarazi et al. (2005).

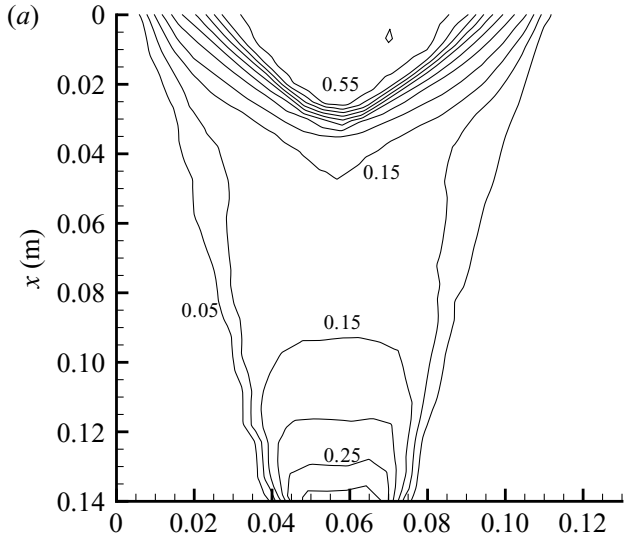

(b)
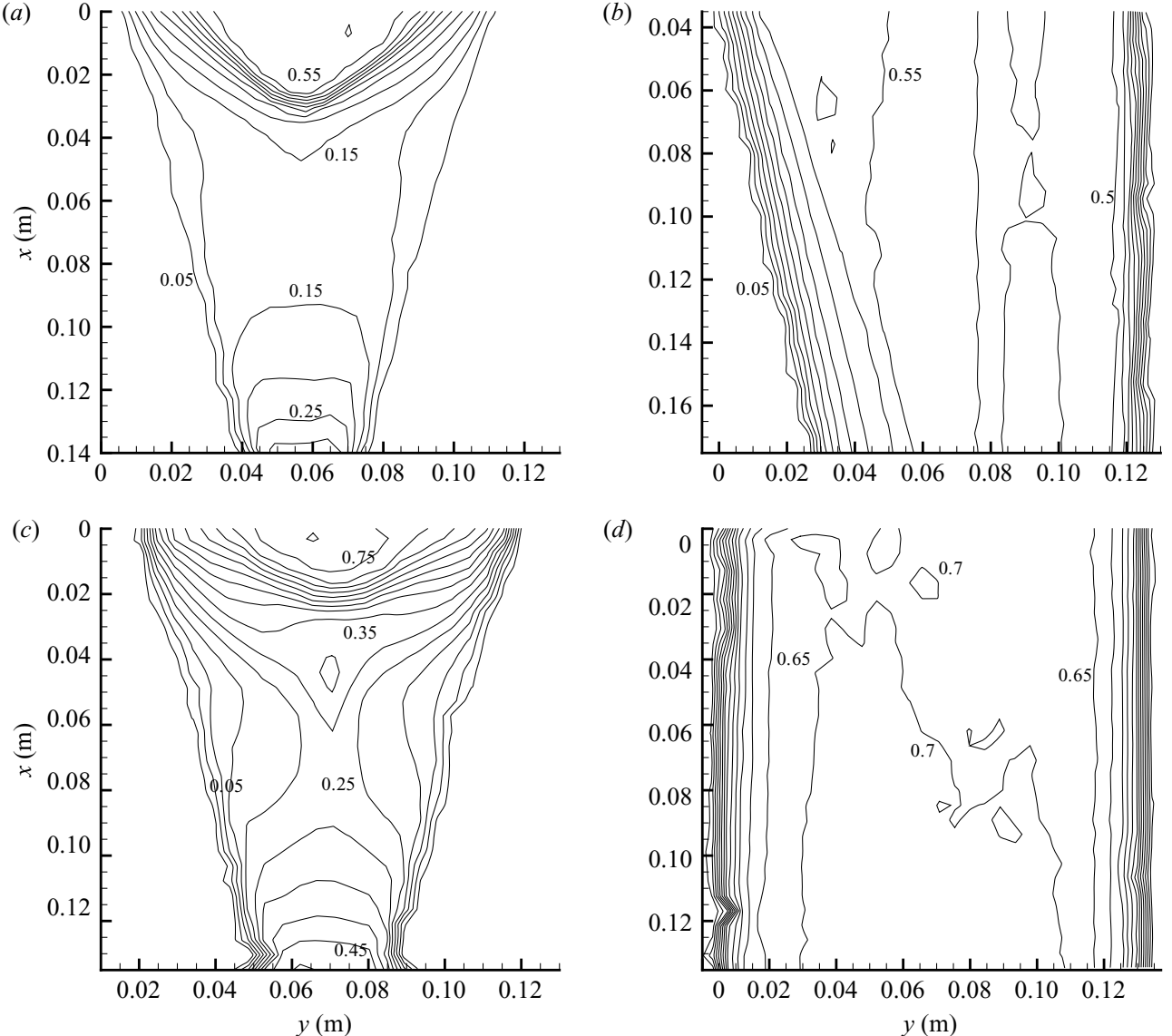

(d)

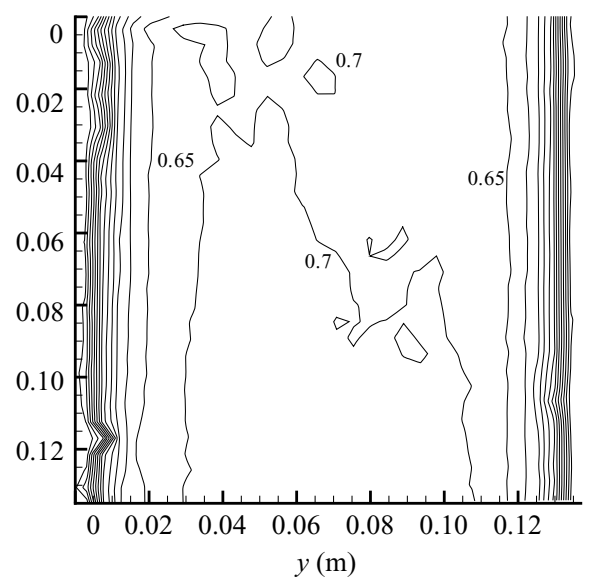

FIGURE 10. Contour lines with steps of $0.05 \mathrm{~m} \mathrm{~s}^{-1}$ are shown for the streamwise velocity of the surface layer, calculated from averaging PIV snapshots over $0.2 \mathrm{~s}$, with small particles (S1) and $F_{0}=3.9$ for $(a)$ a reservoir when $b_{c}=27 \mathrm{~mm}$ and $(b)$ an asymmetric contraction with one wedge; and with large particles (S8) and $F_{0}=2.8$ for (c) a reservoir when $b_{c}=37 \mathrm{~mm}$ and (d) flow without a contraction. 
ratios between the mean and centreline surface velocity: $83 \%$ for $F_{0}=2.8$ and $87 \%$ for $F_{0}=3.9$.

This ratio increases for larger $F_{0}$ as the boundary layer thickness increases for larger Froude numbers (compare the right boundary in figure 10(b) $\left(F_{0}=3.9\right)$ and the boundaries in figure $\left.10(d)\left(F_{0}=2.8\right)\right)$. Thus, the boundary layer is significant in both cases. This complicates the prediction of two-dimensional effects by inviscid theory, as is the case with the angle of the oblique shock shown in figure $10(b)$, because the upstream Froude number reduces near the wall. There are sidewall boundary layers, and the difference between surface and mean velocity, both of which affect the shock angle. In addition, there are frictional and porosity effects. Porosity effects are manifested in the oblique jump which lowers the depth after the shock, recorded at approximately $2 h_{0}$ for the case plotted in figure $10(b)$. These uncertainties may explain why Gray et al. (2003) and Hakonardottir \& Hogg (2005) found differences between their oblique shock predictions (see also Al-Tarazi et al. 2006).

\section{Theoretical analysis}

When the aspect ratios of velocity and length scales normal to and across the chute versus the streamwise direction are small, simplifications can be made by averaging the velocity and volume fraction over a cross-section of the chute and neglecting higherorder aspect ratio effects. Starting with the three-dimensional granular flow equations of Haff (1983), or Lun et al. (1984) without focusing on a particular constitutive stress model, we can extend the asymptotic analysis of Gray et al. (2003) to derive depth- and width-averaged equations. This extension then includes an effective compressibility due to allowable variations in volume fraction. The resulting equations are onedimensional and in the inviscid limit equivalent to the one-dimensional equations governing shallow water and gasdynamics (Baines \& Whitehead 2003; Shapiro 1953). Analysis of similar equations predicts a regime of multiple solutions for shallow flows over a hill (Baines \& Whitehead 2003), and through a contraction (Akers \& Bokhove 2007). For our granular flows, we extend the latter inviscid theory to include effects of friction and porosity, which is essential to explain the experimental results reported so far.

\subsection{Averaged and steady-state equations}

For shallow flows depth-averaging is useful, since the length and velocity scales in the $z$-direction are smaller than the ones in the $x$ - and $y$-directions. As a direct extension of the asymptotic analysis in Gray et al. (2003), including the effects of porosity, the following depth-averaged equations arise in two spatial dimensions with $x$ and $y$ along the plane of the chute:

$$
\begin{aligned}
\frac{\partial}{\partial t}(\alpha h)+\frac{\partial}{\partial x}(\alpha h u)+\frac{\partial}{\partial y}(\alpha h v) & =0, \\
\frac{\partial}{\partial t}(\alpha h u)+\frac{\partial}{\partial x}\left(\alpha h u^{2}+\frac{1}{2} \alpha h^{2} g_{n}\right)+\frac{\partial}{\partial y}(\alpha h u v) & =\alpha h g_{n} \tan \phi-\alpha h g_{n} \mu, \\
\frac{\partial}{\partial t}(\alpha h v)+\frac{\partial}{\partial x}(\alpha h u v)+\frac{\partial}{\partial y}\left(\alpha h v^{2}+\frac{1}{2} \alpha h^{2} g_{n}\right) & =\alpha h g_{n} \tan \phi-\alpha h g_{n} \mu,
\end{aligned}
$$

with depth-averaged volume fraction $\alpha$, and streamwise and crosswise velocity components $u$ and $v$. Instead of the incompressible granular flow equations, their compressible counterparts formed the starting point for this asymptotic analysis. Closure is not obtained because we did not derive a depth-averaged temperature or particle 
volume fraction equation, but restricted ourselves to considering the continuity and momentum equations with a hydrostatic approximation for the pressure. The nondimensional friction coefficient $\mu$ represents all frictional effects.

The next step is to average (3.1) across the chute. The channel walls are at $y=$ $\pm b(x) / 2$, where we use slip flow or a kinematic boundary condition. We assume that flow scales across the chute are much smaller than the ones along the chute. Averaging of (3.1) across the chute gives

$$
\begin{aligned}
\frac{\partial}{\partial t}(\alpha b h)+\frac{\partial}{\partial x}(\alpha b h u) & =0, \\
\frac{\partial}{\partial t}(\alpha b h u)+\frac{\partial}{\partial x}\left(\alpha b h u^{2}+\frac{1}{2} g_{n} \alpha b h^{2}\right) & =\alpha h b g_{n}(\tan \phi-\mu)+\frac{1}{2} g_{n} \alpha h^{2} \frac{\mathrm{d} b}{\mathrm{~d} x},
\end{aligned}
$$

where we have dropped the averaging symbols. The depth- and cross-sectionally averaged volume fraction $\alpha=\alpha(x, t)$, depth $h=h(x, t)$ and streamwise velocity $u=$ $u(x, t)$ depend on $x$ and $t$ only. The width of the chute is $b=b(x)$. The friction force $\mu$ now represents the depth- and cross-sectionally averaged friction. The specific expression for $\mu$ is unknown and generally will depend on $\alpha, u, b$ and $h$. Fluctuational stresses arising as averages of products of fluctuations should be included in $\mu$. Finally, the system (3.2) also follows from a control volume analysis using hydrostatic balance and cross-sectionally and depth-averaged quantities. The derivation assumes that the velocity profile in the $z$-direction is uniform (which is a reasonable approximation for the present granular experiments) and that the ratio of lateral normal and vertical normal stress is equal to one.

Using the continuity equation, the conservative form of the momentum equation can be simplified to

$$
\frac{\partial u}{\partial t}+\frac{\partial\left(u^{2} / 2\right)}{\partial x}=a g_{n}-g_{n} \frac{\partial h}{\partial x} .
$$

The non-dimensional quantity $a$ represents combined effects of gravitational forcing along the chute, porosity, and friction:

$$
a=a_{\alpha}+a_{f}, \quad a_{\alpha}=-\frac{h}{2 \alpha} \frac{\partial \alpha}{\partial x}, \quad a_{f}=\tan \phi-\mu .
$$

In the experiments with a supercritical upstream inflow two possible states downstream were observed, either smooth flow or flow with a strong shock where the flow suddenly becomes subcritical. When $a=0$ equations (3.2) are equivalent to hyperbolic equations such as those in shallow water and compressible gasdynamics. The eigenvalues of (3.2) for $a=0$ and $\alpha$ constant are $\lambda_{ \pm}=u \pm \sqrt{g_{n} h}$, as in the classic shallow water equations. Classical hydraulic or Lavalle-nozzle theory then predicts critical flow with $\lambda_{-}=0$ at the narrowest point of the contraction such that $F_{c}=1$.

The condition $F_{c}=1$ leads to an important curve in the phase diagram of $b_{c}$ and $F_{0}$. The curve is the analytical analogue of the experimental demarcation line $(a)$ in figure 7. We will first derive and generalize this demarcation line by adding the effects of friction and porosity to the classical theory. To start, a relation will be found between the upstream flow - characterized by $h_{0}, u_{0}, \alpha_{0}$ and $b_{0}$ evaluated at the contraction entrance at $x=x_{0}$ - and the flow at the nozzle exit - characterized by $h_{c}, u_{c}, \alpha_{c}$ and $b_{c}$ at $x=x_{c}$. In this formulation, we require the integral functions

$$
A=A_{\alpha}+A_{f} \quad \text { with } \quad A_{\alpha}=\int_{x_{0}}^{x} a_{\alpha} \mathrm{d} x \text { and } A_{f}=\int_{x_{0}}^{x} a_{f} \mathrm{~d} x,
$$


which represent path integration integrals over the previously introduced acceleration terms $a_{\alpha}$ and $a_{f}$. With (3.5), the steady form of the continuity and momentum equations in (3.2) and (3.3) becomes

$$
\frac{\mathrm{d}(\alpha b h u)}{\mathrm{d} x}=0 \quad \text { and } \quad \frac{\mathrm{d}\left(u^{2} / 2+g_{n}(h-A)\right)}{\mathrm{d} x}=0 .
$$

Combining this with the constraint $F_{c}=1$ at the nozzle exit and the uniform and critical state values gives us, for smooth flow, the following three equations:

$$
\begin{aligned}
\alpha_{0} b_{0} h_{0} u_{0} & =\alpha_{c} b_{c} h_{c} u_{c}, \\
\frac{1}{2} u_{0}^{2}+g_{n}\left(h_{0}-A_{0}\right) & =\frac{1}{2} u_{c}^{2}+g_{n}\left(h_{c}-A_{c}\right), \\
u_{c}^{2} & =g_{n} h_{c},
\end{aligned}
$$

with acceleration integrals $A_{0}=A\left(x_{0}\right)=0$ and $A_{c}=A\left(x_{c}\right)$. Substituting (3.7a) into (3.7c), solving for $h_{c}$ and substituting the result into (3.7b), yields the desired relation between $b_{c} / b_{0}$ and $F_{0}$ :

$$
\frac{3}{2}\left(F_{0} b_{0} \alpha_{0} /\left(b_{c} \alpha_{c}\right)\right)^{2 / 3}=1+\frac{1}{2} F_{0}^{2}+Z_{1} \quad \text { with } \quad Z_{1}=A_{c} / h_{0} .
$$

Once the increase in volume fraction $\alpha_{c} / \alpha_{0}$ and the non-dimensional acceleration parameter $Z_{1}$ are known, $b_{c} / b_{0}$ is a function of $F_{0}$. $Z_{1}$ corresponds to the integral acceleration of the critical smooth flow in the contraction from the entrance to the exit.

A second relation is derived next, expressing the occurrence of a steady shock with $x_{s}^{-}=x \uparrow x_{s}$ located just before the shock and $x_{s}^{+}=x \downarrow x_{s}$ just after the shock. We assume $x_{s}=x_{0}$ and $b_{s}=b_{0}$ (later) for clarity, but the derivation holds for arbitrary $x_{s}<x_{c}$. The steady bore front is then at the entrance of the contraction, such that the derived curve is a prediction of the dashed curve $(b)$ in figure 7 for the experimental data. For the flow around the shock and beyond, we have a system of four equations:

$$
\begin{aligned}
\alpha_{0} b_{0} h_{0} u_{0} & =\alpha_{s} b_{s} h_{s} u_{s}=\alpha_{c} b_{c} h_{c} u_{c}, \\
\frac{1}{2} u_{s}^{2}+g_{n}\left(h_{s}-A_{s}\right) & =\frac{1}{2} u_{c}^{2}+g_{n}\left(h_{c}-A_{c}\right), \\
u_{c}^{2} & =g_{n} h_{c},
\end{aligned}
$$

where variables are denoted by subscripts corresponding to their location. From $(3.9 a, b, d)$ we derive expressions for $u_{s}, h_{c}$ and $u_{c}$. We substitute these expressions in (3.9c) to obtain

$$
\left(\alpha_{0} / \alpha_{s}\right) q^{3}=\beta q^{2}-\frac{1}{2}\left(F_{0} b_{0} / b_{s}\right)^{2},
$$

where

$$
\begin{aligned}
& q=\alpha_{s} h_{s} /\left(\alpha_{0} h_{0}\right), \\
& \beta=\frac{3}{2}\left(F_{0} \alpha_{0} b_{0} /\left(\alpha_{c} b_{c}\right)\right)^{2 / 3}-Z_{2} \text { and } Z_{2}=\left(A_{c}-A_{s}\right) / h_{0} .
\end{aligned}
$$

$Z_{2}$ is the non-dimensional acceleration parameter for the lake or reservoir; it corresponds to the acceleration integrated from just after the shock towards the contraction exit. To impose energy dissipation across a granular bore or jump, the (steady) momentum equation in (3.2) is rewritten as

$$
\frac{\mathrm{d}}{\mathrm{d} x}\left(\alpha b h u^{2}+\frac{1}{2} g_{n} \alpha b h^{2}\right)=\alpha b h g_{n} a_{f}+\frac{1}{2} g_{n} \alpha h^{2} \frac{\mathrm{d} b}{\mathrm{~d} x} .
$$


With $x_{s}^{-}=x \uparrow x_{0}$ and $b=b_{0}$ around $x=x_{s}$, the momentum balance across the shock is

$$
\alpha_{0} h_{0} u_{0}^{2}+\frac{1}{2} g_{n} \alpha_{0} h_{0}^{2}=\alpha_{s} h_{s} u_{s}^{2}+\frac{1}{2} g_{n} \alpha_{s} h_{s}^{2}+g_{n} \alpha_{0} h_{0}^{2} Y, \quad Y=\frac{1}{\alpha_{0} h_{0}^{2}} \int_{x_{s}^{-}}^{x_{s}^{+}} \alpha h a_{f} \mathrm{~d} x .
$$

Elimination of $u_{s}$ in (3.14), using $\alpha_{0} b_{0} h_{0} u_{0}=\alpha_{s} b_{s} h_{s} u_{s}$, leads to another third-order polynomial for $q$ :

$$
\frac{\alpha_{0}}{\alpha_{s}} q^{3}=\left(1+2 F_{0}^{2}-2 Y\right) q-2\left(F_{0} b_{0} / b_{s}\right)^{2} .
$$

Subtracting (3.15) from (3.10) results in

$$
\beta=\frac{1+2 F_{0}^{2}-2 Y}{q}-\frac{3\left(F_{0} b_{0} / b_{s}\right)^{2}}{2 q^{2}} .
$$

Next, we introduce two simplifying assumptions: (i) the thickness of the shock is negligible, and (ii) the friction force and therefore $a_{f}$ are continuous. These assumptions imply $b_{s}=b_{0}$ and $Y=0$. Incorporating these consequences into the two expressions (3.12) and (3.16) for $\beta$ implies

$$
\frac{\alpha_{c} b_{c}}{\alpha_{0} b_{0}}=F_{0}\left(\frac{2}{3} Z_{2}+\frac{2+4 F_{0}^{2}}{3 q}-\frac{F_{0}^{2}}{q^{2}}\right)^{-3 / 2} .
$$

After using the physically realizable solution with $q>1$ in (3.15) in (3.17), a complicated but still analytical relation between $b_{c} / b_{0}$ and $F_{0}$ results. For the special case of constant $\alpha$ and $Z_{2}=0$, this relation becomes the inviscid result of Akers \& Bokhove (2007).

In summary, the calculations presented result into two equations, (3.8) and (3.17), leading to predictions of the experimental demarcation lines $(a)$ and $(b)$ in figure 7 , respectively. In the following, we analyse the distinct role played by friction and porosity in our theory, to explain the laboratory observations.

\subsection{Frictional effects}

To assess the role of friction, we will plot isolines of (3.8) and (3.17) in the phase diagram $\left(b_{c} / b_{0}, F_{0}\right)$ for various values of the parameters $Z_{1}$ and $Z_{2}$. Neither parameter is constant, but is instead a function of the flow variables; nevertheless, our extended theory for fixed parameters gives the trends induced by friction and porosity. To investigate frictional effects, we assume the volume fraction to be constant, $\alpha=\alpha_{0}$, in equations (3.8) and (3.17). Each equation gives a curve in the phase diagram, depending on the parameters $Z_{1}$ and $Z_{2}$; these curves are shown in figure 11 as solid and dashed lines, respectively.

These assumptions simplify the analytical expression for the shock curves; (3.15) becomes

$$
(q-1)\left(q^{2}+q-2 F_{0}^{2}\right)=0
$$

with only one relevant $\operatorname{root} q=h_{s} / h_{0}>1$ for $F_{0}>1$, equal to $q=-\frac{1}{2}+\frac{1}{2} \sqrt{1+8 F_{0}^{2}}$, which is substituted into (3.17) to obtain the curves in figure 11 (for $\alpha=\alpha_{0}$ ). The asymptotic behaviour for large $F_{0}$ is

$$
q \sim \sqrt{2} F_{0} \quad \text { and } \quad b_{c} / b_{0}=F_{0} /\left(\frac{2}{3} Z_{2}+\frac{2}{3} \sqrt{2} F_{0}\right)^{3 / 2}
$$

From the latter expression, it is easily seen that positive $Z_{2}$ causes the curve to shift to the left, and vice versa for negative $Z_{2}$, compared to $Z_{2}=0$. Positive $Z_{2}$ corresponds 


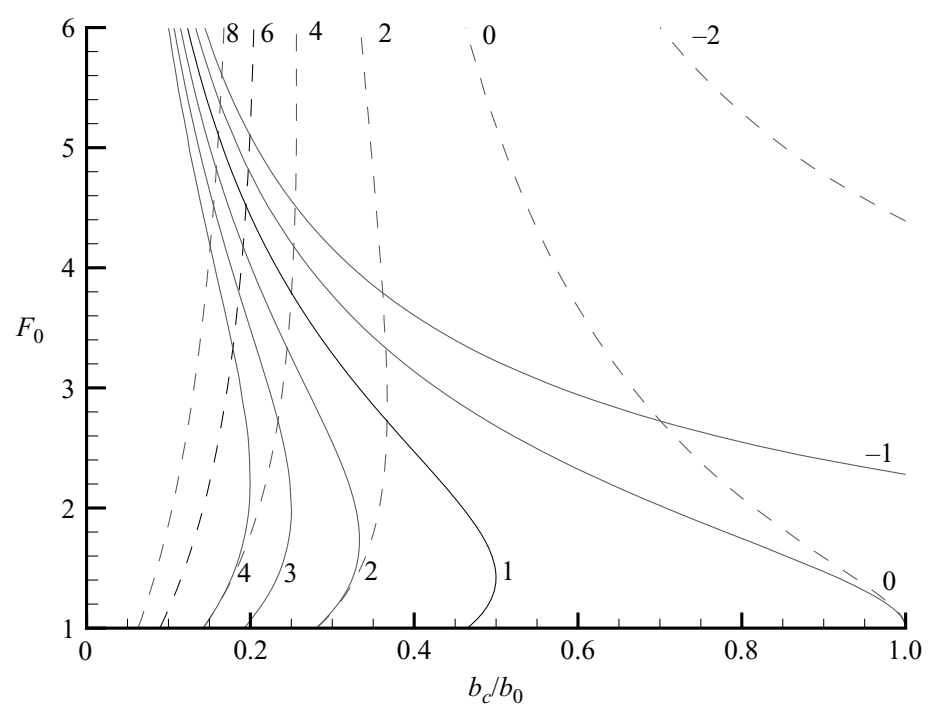

FIGURE 11. Granular 'hydraulic' theory with integral frictional effects predicts two flow types for non-zero friction. Isolines for several values of $Z_{1}$ (critical curves; solid lines) and $Z_{2}$ (shock curves; dashed lines) are shown in the plane $F_{0}$ vs. $b_{c} / b_{0}$.
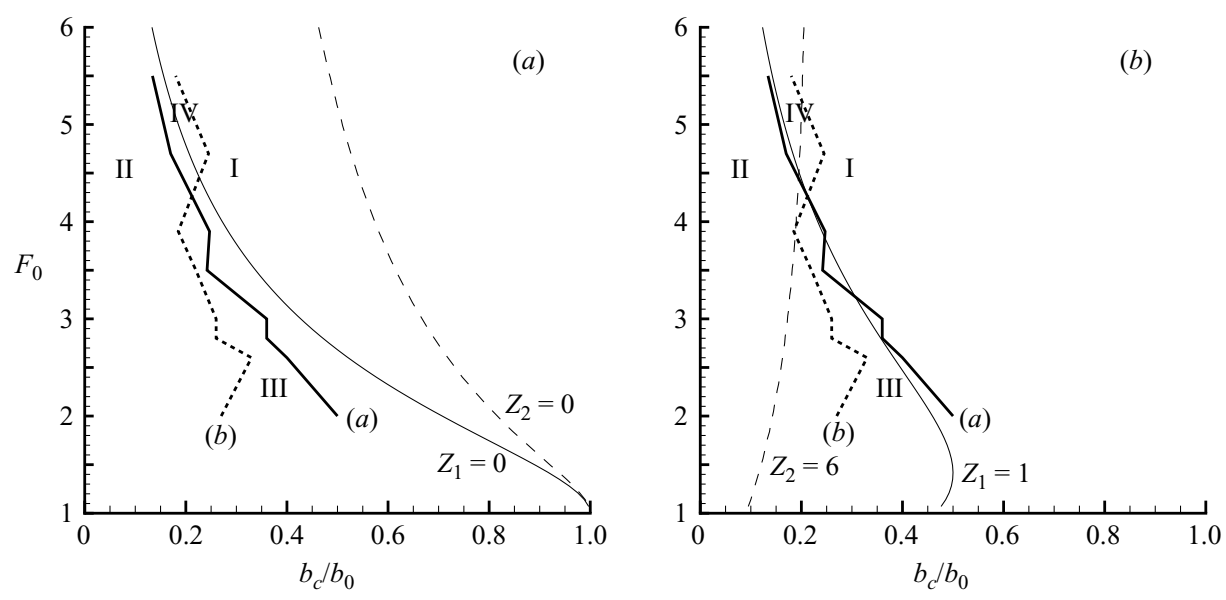

FIgURE 12. Theoretical curves compared with experimental demarcation lines $(a)$ and $(b)$, see figure 7. Critical curves (solid) and shock curves (dashed). (a) Classical theory without friction; (b) extended theory with friction.

to a reduction of friction in the lake/reservoir, while negative $Z_{2}$ corresponds to an increase of friction in the lake/reservoir.

In figure 12 both 'inviscid' and viscous theory are compared with the experimental demarcation lines. In the inviscid case friction parameters are zero, $Z_{1}=Z_{2}=0$, while in the viscous case the friction parameters are selected such that a reasonable agreement with the experimental results is obtained. The 'inviscid' theory would apply at leading order for small changes of the chute width in the contraction and small acceleration effects such that in the reference flow a balance would remain between frictional and gravitational forces. Three striking qualitative differences arise 
between the classical 'inviscid hydraulic' theory and the experimental results; the theory presented indicates how these problems are resolved.

First, where inviscid theory predicts unsteady bores only, the experiments demonstrated the existence of reservoirs with steady bores inside the contraction. Figure 12 clearly shows that the reservoir regime III cannot be predicted by inviscid theory, although it emerges if friction is included. We see that curves shift such that they cross for $F_{0} \approx F_{\text {crit }}=4.0$ and another quadrant emerges below $F_{\text {crit }}$ between the solid and dashed lines for certain values of parameters $Z_{1}$ and $Z_{2}$. In this quadrant, regime III, a steady reservoir exists as a single state with a jump inside the contraction.

Second, according to inviscid theory hysteresis can occur for all $F_{0}>1$ (the large region between the curves $Z_{1}=0$ and $Z_{2}=0$ ). However, in the experiments we observe no hysteretic regime for $F_{0}<4.0$, and the range of nozzle widths $b_{c}$ demarcating the hysteretic regime was much smaller than predicted by inviscid theory for larger values with $F_{0}>F_{\text {crit }}$. However, for certain values of $Z_{1}$ and $Z_{2}$ the lines do cross (here near $F_{0}=4.0$ ), and the regime of hysteresis becomes smaller than in the inviscid case.

Third, according to our experiments supercritical flow occurs for lower values of $b_{c}$ than given by inviscid theory: compared to the experimental results the solid inviscid curve $Z_{1}=0$ is too far to the right. However, using a positive value of $Z_{1}=1$ the curve shifts to the left and becomes closer to the solid demarcation line plotted from the experiments.

Apparently, positive values of $Z_{i}(i=1,2)$ are required to obtain reasonable agreement between theory and the experiments with glass beads. Positive values of $Z_{i}$ are equivalent to positive acceleration integrals, which means that in the reservoir the friction coefficient $\mu$ is smaller than in the flow upstream. The flow upstream is supercritical, but in the reservoir the Froude number can be much lower than one. For a reservoir which fills the contraction, $Z_{2} \approx 6$, and in the reservoir the Froude number $F$ has dropped below one. The friction parameter is much lower for critical flow, $Z_{1} \approx 1$ and for such a flow $1<F<F_{0}$ in the contraction. These findings indicate that the friction coefficient $\mu$ for spherical particles in a contraction decreases with $F$. Indeed, in most friction laws friction is lower when $F$ is reduced (see $\S 5$ ), suggesting that $Z_{2}$ should be positive.

According to the theory presented each upstream travelling bore becomes steady at some point, provided the friction $\mu$ is reduced in the lake. The latter implies $Z_{2}>0$, which corresponds with our observations. Assuming friction reduction in the lake, $Z_{2}$ increases monotonically with the length $L_{l}=x_{c}-x_{s}$ of the lake. To show that a shock eventually stops moving, the upper bound of this lake length $L_{l}$ is estimated from the asymptotic equations (3.19) by

$$
L_{l}=\frac{3}{2} h_{1}\left(F_{1} b_{1} / b_{c}\right)^{2 / 3} /(\tan \phi-\mu),
$$

using the approximation

$$
Z_{2}=\frac{1}{h_{1}} \int_{x_{s}}^{x_{c}} a \mathrm{~d} x \approx L_{l}(\tan \phi-\mu) / h_{1}
$$

with $h_{1}, F_{1}$ and $b_{1}=b_{0}$ the depth, Froude number and channel width at $x=x_{1}=x_{s}^{-}$; $\phi$ is the angle of inclination and $\mu$ the approximately constant friction in the lake. For uniform upstream flow or $x_{s}=x_{0}$, we have $h_{1}=h_{0}$ and $F_{1}=F_{0}$.

In the experiments reported here, the flow condition before the lake or contraction was uniform or slightly accelerating $(\$ 2)$. However, suppose the upstream flow experiences a constant acceleration $\eta$, or deceleration for negative $\eta$. From the 
definition of $A$ and $Z$, it is apparent that $Z$ should then be corrected using $\eta\left(x-x_{0}\right) / h_{0}$, with $x_{0}$ as the beginning of the contraction. In the parameter plane, this yields an extra left-shift for accelerating and a right-shift for decelerating chute flow. The main effect of acceleration or deceleration upstream of the contraction is that the local Froude number changes. A small acceleration present in an experiment before the contraction is therefore captured because the Froude number $F_{0}$ is measured at the entrance of the contraction. In $\S 2.1$, we found $0.1 \mathrm{~m} \mathrm{~s}^{-2}$ as the upper bound of this acceleration, and measured a contraction length $L_{c}=0.20 \mathrm{~m}$ and depth $h_{0}=2 \mathrm{~mm}$. Hence the effect of the (scaled) acceleration along the contraction is approximately $\Delta Z_{1}=\Delta Z_{2}=0.1 L_{c} /\left(h_{0} g_{n}\right)=1$. Figure 11 shows that these contributions do not alter the essentials of the theory because both demarcation lines in the $b_{c}, F_{0}$ phase plane simply shift by the same value $\Delta Z$.

Whereas for the spherical glass beads we found positive values of $Z_{i}$, for poppy seeds we found that negative values $Z_{2} \approx-2$ and $Z_{1}<-1$ are required to obtain reasonable agreement between theory and experiment. This indicates that changes in shape and density of particles can alter the behaviour of $\mu$ inside the contraction: for spherical particles friction reduces while for poppy seeds friction increases, where $F<F_{0}$. None of the friction models above is able to explain this fundamentally different behaviour of spherical and the lighter, non-spherical particles. For these non-spherical particles, the increase of particle volume fraction in the contraction seems to influence the friction coefficient $\mu$ more than for spherical particles. Nonspherical particles roll less and an increase of the friction may be due to 'locking' of the particles which increases the volume fraction.

\subsection{Effects of porosity}

To assess the role of porosity, we plot isolines of (3.8) and (3.17) in the phase diagram $\left(b_{c} / b_{0}, F_{0}\right)$ for various values and simplifications of $\alpha / \alpha_{0}$. From the granular experiments, we know that porosity changes are significant. The typical particle volume fraction measured in a lake is $0.6(\S 2)$ which, together with the measured values for $\alpha_{0}$ in table 1 , means that $\alpha_{s} / \alpha_{0}$ varies between 1.3 and 2.4. However, for supercritical flows the ratio $\alpha_{c} / \alpha_{0}$ is expected to be much lower, say 1.3. When shocks occur, we for the moment adopt the simplifying assumption that the volume fraction only increases through the shock and stays constant in the lake and take $\alpha_{s}=\alpha_{c}$. Then we calculate the modification of the demarcation lines, using (3.8), and (3.15) and (3.17). Two sets of isolines for various values of $\alpha_{c} / \alpha_{0}$ are shown in figure 13 for $Z_{1}=Z_{2}=0$. Frictional effects and gradients of $\alpha$ in $A$ are thus neglected in figure 13 . The results displayed suggest that if we take non-zero values of $Z$ the isolines of $\alpha_{c} / \alpha_{0}$ retain their order and approximately their separation.

For these realistic values of $\alpha_{c} / \alpha_{0}$, less than 2 for the shock curve and about 1.3 for the critical curve, it is clear from figure 13 that porosity, unlike friction, is unable to explain the differences between experiment and inviscid theory by itself. Yet from the shifts predicted in figure 13, we conclude that porosity and friction reinforce one another for the shock curve. Both effects cause the curves to shift to the left, which again supports $Z_{2}>Z_{1}$.

Apart from the ratio $\alpha_{c} / \alpha_{0}$ there is another porosity effect on the curves, namely the term $A_{\alpha}$ in (3.5). This term does not alter the above-mentioned conclusions. For the shock curve, $A_{\alpha}$ approximately vanishes given the (reasonable) assumption of constant and nearly maximum volume fraction in the entire lake. For the critical curve, we assume a gradual increase from $\alpha_{0}$ to $\alpha_{c}$ in the contraction. For $\alpha_{c} / \alpha_{0}=1.3$ and typically $h_{c}=3 h_{0}$ for granular supercritical flows, we approximate the contribution 


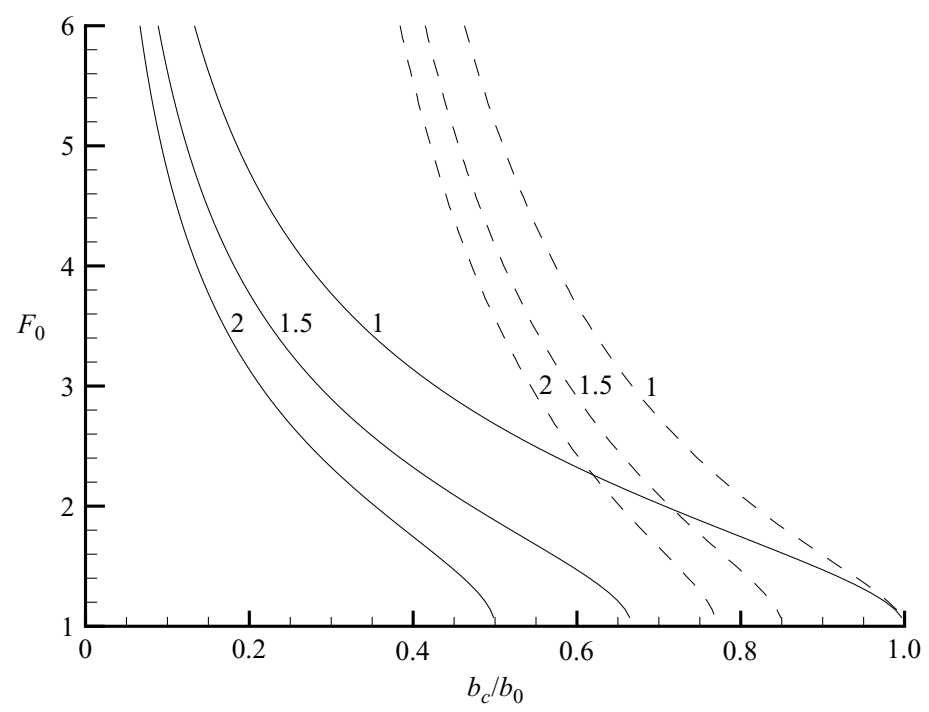

FIGURE 13. The effects of porosity are displayed in the plane $F_{0}$ vs. $b_{c} / b_{0}$ as isolines for several values of $\alpha_{c} / \alpha_{0}$ in the absence of friction such that $A=0$; critical curves (solid) and shock curves (dashed).

of $A_{\alpha} / h_{0}$ to $Z_{1}$ by

$$
-\frac{\frac{1}{2}\left(h_{0}+h_{c}\right)\left(\alpha_{c}-\alpha_{0}\right)}{2 \times \frac{1}{2}\left(\alpha_{0}+\alpha_{c}\right) h_{0}} \approx-0.3 .
$$

This means that the influence of $A_{\alpha}$ on the critical curve is small.

Finally, we explored the influence of porosity on the shock speed with some additional experiments. Material and parameters correspond to S0. When the granular flow is entirely blocked in the middle of the chute for $\phi=15.5^{\circ}$ a granular bore develops, see figure $1(c)$. We consider a constant upstream state with values $u_{0}, h_{0}, \alpha_{0}$ and a quiescent state with $u_{+}=0, h_{+}, \alpha_{+}$downstream of the bore. The jump relations follow from (B 4) and $u_{+}=0$ in Appendix B. This yields the dimensional bore speed

$$
S_{\alpha}=-\sqrt{\frac{g_{n}}{2} \frac{\alpha_{0} h_{0}}{\alpha_{+} h_{+}} \frac{\left(\alpha_{+} h_{+}^{2}-\alpha_{0} h_{0}^{2}\right)}{\left(\alpha_{+} h_{+}-\alpha_{0} h_{0}\right)} .}
$$

Given $h_{0}, \alpha_{0}, h_{+}$and $\alpha_{+}$we can predict $S_{\alpha}$, and $u_{0}$ from (B 2). For constant $\alpha$, (3.23) reduces to the granular bore speed $S=\lim _{\alpha_{0} \rightarrow \alpha_{+}} S_{\alpha}$ used by Gray et al. (2003). We did the experiment three times and the results of all three were reasonably accurate. Measurements are $h_{0}=2.1 \pm 0.1 \mathrm{~mm}, h_{+}=8.5 \pm 0.2 \mathrm{~mm}$ and $S_{P I V}=0.073 \pm 0.001 \mathrm{~m} \mathrm{~s}^{-1}$. For constant $\alpha$, the prediction by (3.23) results in $S=0.11 \pm 0.005 \mathrm{~m} \mathrm{~s}^{-1}$, which is $1.5 \pm 0.1$ times too large. To include porosity effects, we take for the upstream porosity $\alpha_{0}=0.36 \pm 0.06$, obtained from the lowest three values in figure 9. Downstream the material is at rest, such that we have the maximum packing of spheres, $\alpha_{+}=0.64$. Using these values in (3.23) gives the prediction, $S_{\alpha}=0.079 \pm 0.012 \mathrm{~m} \mathrm{~s}^{-1}$, which is $1.08 \pm 0.16$ times the measured value. We conclude from these sets of experiments that porosity is important, at least when the flow coming into the bore is thin, in this case 4 to 5 particle diameters $d$ (see also Appendix B). 


\section{Simulations}

In this section, we consider three-dimensional simulations of granular layers through a contraction on a downhill slope. First, we will investigate whether discrete-particle simulations are able to predict the experimental observations presented, and in particular the occurrence of a reservoir state. Second, we will precisely quantify the effects of friction and porosity through the simulations and seek further confirmation of the hydraulic theory presented. The simulations concern the dynamics of discrete particles, calculated from a soft-sphere discrete-particle model, similar to the model described in detail by Van der Hoef et al. (2006) and applied to uniform granular flow on inclined planes by Silbert et al. (2003). These models solve the Lagrangian equations for spherical particles with a diameter $d$, based on Newton's laws for the velocity and the angular velocity. The contact forces between the particles are calculated using a so-called linear spring/dash-pot model (Cundall \& Strack 1979). For each pair of particle contacts, the normal displacement between the two particles, say $a$ and $b$, is calculated as leading to a normal interaction force $\boldsymbol{F}_{n, a b}$, directed along the normal $\boldsymbol{n}_{a b}$,

$$
\boldsymbol{F}_{n, a b}=-\left(k_{n}\left(d-\left|\boldsymbol{r}_{a}-\boldsymbol{r}_{b}\right|\right)+\eta_{n} \boldsymbol{v}_{a b} \cdot \boldsymbol{n}_{a b}\right) \boldsymbol{n}_{a b} \quad \text { if } \quad\left|\boldsymbol{r}_{a}-\boldsymbol{r}_{b}\right|<d,
$$

where $\boldsymbol{r}_{a}$ and $\boldsymbol{r}_{b}$ are the location vectors of the particles, $\boldsymbol{v}_{a b}$ the relative velocity of $a$ and $b$, and $k_{n}$ and $\eta_{n}$ model constants. The spring stiffness $k_{n}$ is chosen to be $100 \mathrm{~N} \mathrm{~m}^{-1}$. This is relatively low, but nevertheless sufficiently high for the present purposes. The maximum overlap of a soft sphere as a function of time fluctuated between $0.01 d$ and $0.02 d$. The damping coefficient $\eta_{n}$ is defined as

$$
\eta_{n}=-2 \sqrt{\frac{k_{n}}{\left(\pi^{2}+(\ln e)^{2}\right)\left(m_{a}^{-1}+m_{b}^{-1}\right)}} \ln e,
$$

where $e$ is the normal restitution coefficient, taken as equal to 0.97 , a realistic value for glass beads (Goldschmidt, Beetstra \& Kuipers 2004). The mass of body $a$ involved in the contact is denoted by $m_{a}$, which equals either the mass of a single particle or infinity if the body $a$ represents a wall. The magnitude of the tangential force is modelled by $\mu_{t}\left|\boldsymbol{F}_{n}\right|$, which resembles a Coulomb friction law. The friction coefficient $\mu_{t}$ is taken equal to $\tan \phi=0.344$, where $\phi=19^{\circ}$ (table 1). It is close to the internal friction angle mentioned by Hakonardottir \& Hogg (2005). The interaction with air is neglected in the computational model, since its effect is assumed to be small; an upper bound for the drag force exerted by the surrounding air on a $1 \mathrm{~mm}$ glass bead is estimated to be about $6 \%$ of the tangential gravitional force. The estimate is based on the standard nonlinear drag law for a single particle moving with a velocity of $0.6 \mathrm{~m} \mathrm{~s}^{-1}$ in stagnant far-field surroundings. The physical drag force will be considerably lower than this upper bound for particles below the surface.

In our simulations, we focused on the experimental set S8 of large particles. We simulated the chute flow through a contraction for an inclination angle of $19^{\circ}$ and $g=9.8 \mathrm{~m} \mathrm{~s}^{-2}$. We used a uniform $d=1 \mathrm{~mm}, \rho_{p}=2470 \mathrm{~kg} \mathrm{~m}^{-3}$, and investigated flows for several contraction widths $b_{c}$. The velocity imposed at the inflow consisted of a constant mean plus time-dependent three-dimensional random perturbations of $2 \%$. The height of the inflow was approximately the height of the gate at the top of the chute in the experimental set-up. The value of the mean inflow velocity was $0.17 \mathrm{~m} \mathrm{~s}^{-1}$. The inflow volume fraction was high and determined by matching computational and experimental mass fluxes $\left(0.29 \mathrm{~kg} \mathrm{~s}^{-1}\right)$. For reasons of computational efficiency, we used a relatively short chute length of $0.70 \mathrm{~m}$ to limit the maximum number of particles 


$\begin{array}{clcc}b_{c} & \text { Curve } & \text { Time }(\mathrm{s}) & \text { No. of particles } \\ 130 \mathrm{~mm} & \text { dashed } & 7.0 & 284000 \\ 70 \mathrm{~mm} & \text { dotted } & 6.3 & 304000 \\ 50 \mathrm{~mm} & \text { dash-dotted } & 7.6 & 323000 \\ 40 \mathrm{~mm} & \text { solid } & 10.8 & 378000\end{array}$

TABLE 2. Overview of discrete-particle simulations for various nozzle widths $b_{c}$. Note that $b_{c}=0.13 \mathrm{~m}$ corresponds to the case without a contraction.

to about 400000 . The contraction was placed between $x=0.30 \mathrm{~m}$ and $0.50 \mathrm{~m}$. The equations were integrated sufficiently long to let the flow evolve to a quasi-steady state. In particular, for the smallest value of $b_{c}$ the integration time was long, $10.8 \mathrm{~s}$, corresponding to $2.2 \times 10^{5}$ time steps for the fourth-order four-stage Runge-Kutta method. In each simulation time averaging was performed to obtain statistics. They did not depend significantly on the length of the time interval of averaging, which was at least $1.0 \mathrm{~s}$ for each case. An overview of parameters for the simulations is given in table 2, including the final time and the number of particles in the system. We will show that the simulation for $b_{c}=40 \mathrm{~mm}$ produced a reservoir, while the simulations with higher values of $b_{c}$ led to supercritical flow.

To calculate statistics we need to define appropriate averaging operators. The time-average of a three-dimensional field $u$ is defined by

$$
\langle\alpha u\rangle_{t}=\frac{\pi d^{3}}{6 \tau \Delta x \Delta y \Delta z} \int_{t-\tau}^{t} \sum_{i} u_{i} \mathrm{~d} t
$$

where the sum is taken over all particles with $x y z$-coordinates of their centres inside a local cube with ribs $\Delta x=\Delta y=2 \mathrm{~mm}$ and $\Delta z=1 \mathrm{~mm}$ around the point $(x, y, z)$. The averaged quantity is a piecewise continuous field. The average of $u$ is now defined by

$$
[u]_{t}=\frac{\langle\alpha u\rangle_{t}}{\langle\alpha\rangle_{t}} .
$$

The denominator is obtained by evaluating (4.3) for $u=1$.

The cross-sectional average is defined by

$$
[u]_{t y z}=\frac{\langle\alpha u\rangle_{t y z}}{\langle\alpha\rangle_{t y z}}, \quad\langle\alpha u\rangle_{t y z}=\frac{1}{b h} \int_{0}^{L_{2}} \int_{-\frac{1}{2} b}^{\frac{1}{2} b}\langle\alpha u\rangle_{t} \mathrm{~d} y \mathrm{~d} z .
$$

Note that we are allowed to extend the outer integration to a fixed $L_{2}>h$, the computational depth of the domain, since $\alpha$ occurs in the integrand. In this way, we can appropriately handle the free boundaries in the flow once we have appropriate definitions for the extent of the flow, $h$ and $b$. An isolated depth-average (transversal average) is obtained by omitting $b(h)$ and the integral over $y(z)$ and the symbol $y$ (z) in the subscript.

To define the depth we introduce

$$
\hat{z}(x, y, t)=\frac{1}{\tau} \int_{t-\tau}^{t} \max _{i}\left\{z_{i}\right\} \mathrm{d} t,
$$

where the maximum is taken over the $z$-coordinate of particles centres with $x y$ coordinates inside a local square of $\Delta x=\Delta z=2 \mathrm{~mm}$ around $(x, y)$. The time integral 

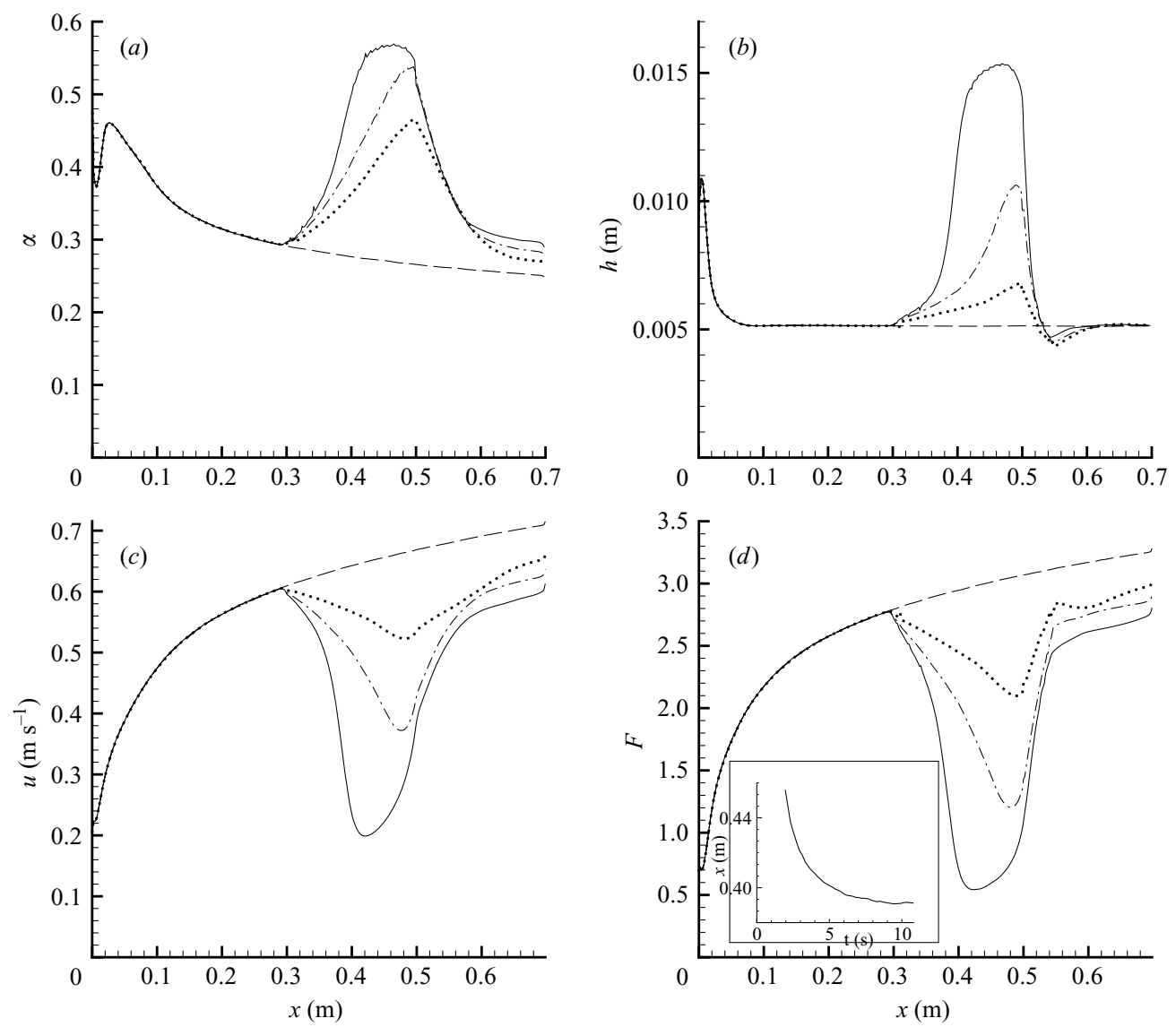

FiguRE 14. Cross-sectionally averaged steady-state profiles for $b_{c}=130$ (dashed), 70 (dotted), 50 (dash-dotted) and $40 \mathrm{~mm}$ (solid). The contraction is between $x=0.3$ and $0.5 \mathrm{~m}$. (a) Particle volume fraction, $(b)$, depth, $(c)$ streamwise velocity and $(d)$ Froude number. The inset in $(d)$ shows the $x$-value for which $F=1$ as a function of time and thus represents the evolution of the upstream boundary of the reservoir.

is over an interval of $\tau=0.04 \mathrm{~s}$. Then we define the depth of the layer by

$$
h(x, y, t)=\hat{z}+\frac{1}{2} d+\sqrt{\widehat{z}^{2}-\hat{z}^{2}} .
$$

In the free jet region the width $b$ for a given $x$ and $z$ is estimated by counting the number of grid cells with non-zero volume fraction. The value of $\tau$ is sufficiently short to follow adequately the temporal behaviour in the transient regime. When the flows became steady we tried larger values of $\tau$ (up to $0.8 \mathrm{~s}$ ), but we did not observe substantial differences with $\tau=0.04 \mathrm{~s}$.

The cross-sectional averages of depth, volume fraction, velocity and Froude number are shown in figure 14 for all four simulations. At the contraction entrance $(x=0.3 \mathrm{~m})$, we computed $u_{0}=0.61 \mathrm{~m} \mathrm{~s}^{-1}, h=4.7 \mathrm{~mm}, \alpha_{0}=0.31$ and $F_{0}=2.9$. These values are very close to the experimental values for S8 listed in table 1 . We observed that the computed flow without a contraction is still slightly accelerating beyond $x=0.30 \mathrm{~m}$, which is most clear from the streamwise velocity in figure $14(\mathrm{c})$. The acceleration decreases with $x$ and the average acceleration between $x=0.3$ and $0.5 \mathrm{~m}$ is $0.20 \mathrm{~m} \mathrm{~s}^{-2}$. 
We note from the Froude number displayed in figure $14(d)$ that the flow is clearly supercritical for $b_{c} \geqslant 50 \mathrm{~mm}$, but for $b_{c}=40 \mathrm{~mm}$ we see a subcritical region with a length of $0.111 \mathrm{~m}$. The latter case corresponds to a steady reservoir. The bore front is defined to be at the location where $F=1$ in the contraction. The inset of figure $14(d)$ shows the location of the bore front as a function of time. It formed around $t=2 \mathrm{~s}$ at $x=0.47 \mathrm{~m}$ and converged at $t \approx 17 \mathrm{~s}$. At $t=2 \mathrm{~s}$ the bore velocity was about $32 \mathrm{~mm} \mathrm{~s}^{-1}$, at $t \approx 10 \mathrm{~s}$ about $1 \mathrm{~mm} \mathrm{~s}^{-1}$ and at $t \approx 17 \mathrm{~s}$ approximately 0 .

That a reservoir appeared for $b_{c}=40 \mathrm{~mm}$ is consistent with the experimental data since for the experimental set S8 we observed a reservoir for $b_{c}=40,38,37$ and $35 \mathrm{~mm}$. For $b_{c}=37 \mathrm{~mm}$ the reservoir length was measured and found to be $0.12 \mathrm{~m}$. The shortest reservoir was found for the largest $b_{c}$ (see figure 7 for $F_{0}=2.8$ and figure 10c). Thus, the simulated reservoir length of $0.111 \mathrm{~m}$ for $b_{c}=40 \mathrm{~mm}$ is in line with the available experimental data; reservoir length and $b_{c}$ are both within $10 \%$ of the measured values. The depth of the simulated reservoir at $b_{c}=40 \mathrm{~mm}$, about $15 \mathrm{~mm}$ according to figure $14(b)$, is the same as we measured in the experiment for $b_{c}=37 \mathrm{~mm}$. The maximum volume fraction in the reservoir is 0.57 (figure $14 a$ ), which is within the measurement error of the measured value of 0.52 (with error less than $13 \%$, see table 1 ). The compression of the soft particles in the simulation leads to a slight overprediction of $\alpha$. By monitoring the maximum compression mentioned before, we estimate that the calculated $\alpha$ should be corrected to $0.57 /\left(1.015^{3}\right)=0.54$.

Contour plots of depth-averaged velocity components, depth, and volume fraction are shown in figure 15. The structure of the simulated and experimental streamwise velocity component is similar (compare figures $15 a$ and $10 c$ ). The depth-averaged calculated values should be lower because the PIV-result corresponds to the surface velocity. It appears that the difference between PIV surface velocity and depthaveraged velocity is about $10 \%$. The granular temperature $T$ is defined by

$$
T=\frac{1}{3}\left([\boldsymbol{u} \cdot \boldsymbol{u}]_{t}-[\boldsymbol{u}]_{t} \cdot[\boldsymbol{u}]_{t}\right)
$$

The depth-average of the granular temperature is the integral over $z$ of $\langle\alpha\rangle_{t} T$ divided by $h\langle\alpha\rangle_{t z}$. It is shown in figure $15(f)$. The value upstream of the contraction corresponds to an average fluctuation intensity of $\sqrt{0.0025}=0.05 \mathrm{~m} \mathrm{~s}^{-1}$, approximately $10 \%$ of $u_{0}$. The flow is very quiet in the reservoir, as the fluctuation level is much lower there.

The forces in the discrete-particle model consist of gravitational acceleration and the surface contact forces between particles and between particles and walls. Thus a single particle experiences an acceleration of $\tan \phi+a_{\text {contact }}$, where $a_{\text {contact }}$ is the sum of the contact forces felt through neighbouring particles or walls. To validate the theory developed in the previous section, we consider the streamwise and cross- and depthaveraged components of these terms in figure 16. Considering the contact friction forces in more detail, we observe that in the reservoir (see figure $16 a$ for the case $b_{c}=40 \mathrm{~mm}$ in the interval $0.39 \leqslant x \leqslant 0.50 \mathrm{~m}$ ) the absolute value of the friction due to contact forces decreases dramatically, which confirms the theory of the previous section.

To obtain more insight, we calculated the integrated acceleration $A=\int_{x_{0}}^{x} a \mathrm{~d} x$ defined in $\S 3$. It is, however, not simply the integral over $\tan \phi+a_{\text {contact }}$, since $a_{\text {contact }}$ also contains the integral over the granular pressure force. Hence, using the hydrostatic pressure in (3.3), we rewrite

$$
A=h+\int_{x_{0}}^{x}\left(\tan \phi+a_{\text {contact }}\right) \mathrm{d} x,
$$



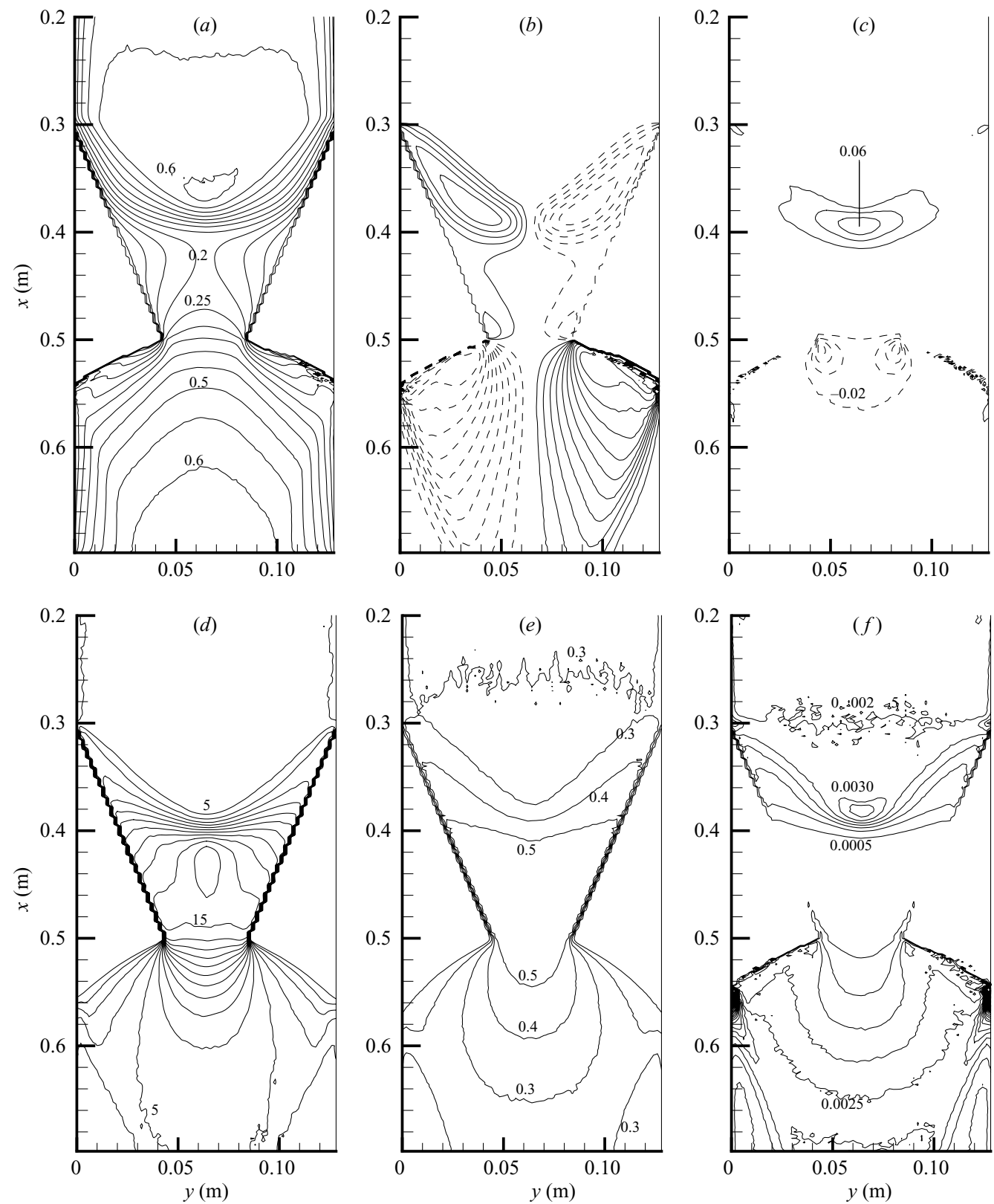

FIGURE 15. Depth-averaged steady-state contour plots for $(a)$ streamwise velocity $u,(b)$ spanwise velocity $v,(c)$ normal velocity $w,(d)$ depth $h,(e)$ particle volume fraction $\alpha$ and $(f)$ granular temperature $T$. Negative contours are dashed. Contour increments are $(a) 0.05 \mathrm{~m} \mathrm{~s}^{-1}$, (bc) $0.02 \mathrm{~m} \mathrm{~s}^{-1},(d) 1 \mathrm{~mm},(e) 0.1$, and $(f) 0.0005 \mathrm{~m}^{2} \mathrm{~s}^{-2}$.

where $x_{0}=0.30 \mathrm{~m}$ denotes the entrance of the contraction in the simulations. The quantity $A / h_{0}$ is shown in figure $16(b)$ for the four simulated flows. The strong increase of $A / h_{0}$ in the reservoir region supports the friction theory in $\S 3$. The normalization with $h_{0}$ allows us to compare $\left(A_{c}-A_{s}\right) / h_{0}$, the difference of $A / h_{0}$ in this figure between $x_{c}=0.5 \mathrm{~m}$ and $x_{s}=0.39 \mathrm{~m}$ for $b_{c}=40 \mathrm{~mm}$, with the $Z$-values 

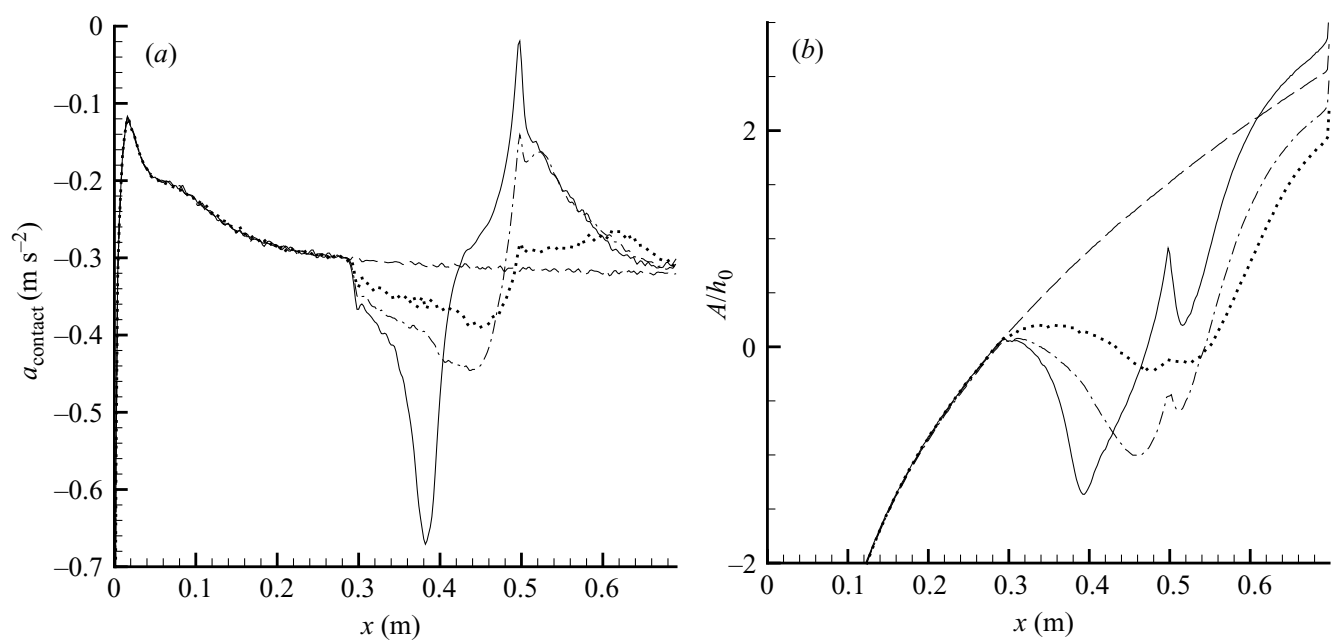

FiguRE 16. Cross- and depth-averaged steady-state profiles for $b_{c}=130$ (dashed), 70 (dotted), 50 (dashed-dotted) and $40 \mathrm{~mm}$ (solid). The contraction is between $x=0.3$ and $0.5 \mathrm{~m}$. (a) Acceleration term $a_{\text {contact }}$ due to contact forces. $(b)$ Integrated acceleration term $A / h_{0}$.

discussed in the previous section. We observe that $A / h_{0}$ increases to 2 in the reservoir region $0.39 \leqslant x \leqslant 0.50 \mathrm{~m}$ for $b_{c}=40 \mathrm{~mm}$. Indeed, the value $A / h_{0}=2$ is between $Z_{1}=1$ and $Z_{2}=6$. The latter values correspond to the theoretical qualitative prediction of the reservoir regime, as plotted in figure $12(b)$. In the first part of the contraction the friction does not become weaker but stronger, however, as $A / h_{0}$ reduces just before the reservoir (solid curve). For the smooth flow $\left(b_{c} \geqslant 50 \mathrm{~mm}\right.$, dashed-dotted curve), the friction is increased in the entire contraction, since $A / h_{0}$ decreases between $x_{0}=0.3 \mathrm{~m}$ and $x_{c}=0.5 \mathrm{~m}$. This surprising result will be clarified in $\S 6$ where we analyse several constitutive laws used for depth-averaged models in the literature. The isolated effect of extra friction would cause a rightward shift for the critical curve. For the critical curve, we therefore infer that the left-shift due to porosity appears to be stronger than the right-shift due to extra friction, such that a relatively small net left-shift results.

The crucial point in the theory explaining the reservoir is that due to friction the shock curve was able to shift to the left from the critical curve. The extended hydraulic theory, in combination with our observations, showed that the emergence of a reservoir state was caused by a reduction of friction in the contraction. This led to a larger left-shift $\left(Z_{1}>0\right)$ of the shock curve stemming from clasical, inviscid hydraulic theory than the left shift $\left(Z_{2}>0\right)$ of the critical curve, such that $Z_{2}-Z_{1}>0$. To verify the latter, we calculate the difference between $A / h_{0}$ for the case $b_{c}=40 \mathrm{~mm}$ and for $b_{c}=50 \mathrm{~mm}$ at $x=x_{c}=0.50 \mathrm{~m}$ in our simulations. According to figure $16(b)$, this value $\sim Z_{2}-Z_{1}$ is 1.0 , which is positive. We conclude therefore that both curves experience a shift to the left due to porosity effects corrected with an additional shift to the right because the granular flow appears to experience an increase of friction in the first part of the contraction between $0.3<x<0.4 \mathrm{~m}$. This increase of friction before the reservoir combined with a reduction of friction in the reservoir illustrates the complexity of modelling the constitutive friction laws for granular flows.

From figure $16(b)$, we also see that the acceleration of the flow without a contraction corresponds to $\Delta Z \approx 1$. As argued in $\S 3$, constant accelerations of the flow without a contraction do not alter essentials of the theory, since both demarcation lines in the $b_{c}, F_{0}$ phase plane shift by the same value $\Delta Z$. 
The calculation is also used to find the magnitude of two terms that were discarded in the theoretical derivation. The first term is $A_{\alpha}$, which is a correction due to the main effect of porosity represented by $\alpha_{c} / \alpha_{0}$. The second term is an acceleration integral of fluctuational stresses arising from the cross- and depth-averaged equations, which were assumed to be included in $\mu$. Both terms appear to be negative and after normalization with $h_{0}$ they lead to corrections of the acceleration integrals varying between -0.5 and -0.2 for several $b_{c}$.

\section{Discussion of rheology}

In this section, we discuss the rheology of the following existing friction models applicable to smooth planes: the Coulomb model, the kinetic-collisional model of Johnson et al. (1990), the Savage-Hutter (1991) friction model, and the model of Louge \& Keast (2001). We consider the discrete-particle simulations as references, because they correspond rather well with the laboratory observations and contain the required detailed information on the granular stresses and their averages. Hence, the numerical simulations are used to calculate 'actual' pressure and other fields, which are then used as input for the theoretical friction models proposed in the literature. The predicted stresses resulting from these friction models are then validated against the actual (depth- and width-averaged) granular stresses in our simulations. Such a validation is called a priori testing because no partial differential equations incorporating the constitutive models have to be solved.

First, the most simple frictional model is the Coulomb model (as used by, among others, Gray et al. 2003), in which the ratio between tangential and normal stresses is constant. Estimating the normal stress at the bottom as the hydrostatic pressure, $\rho_{p} \alpha g_{n} h$, we find $\mu=\tan \delta$. However, the Coulomb friction model is too simple to explain the steady granular reservoir since it is not able to reduce the friction in a contraction significantly. A slight reduction is obtained because the friction coefficient is formally multiplied with $u /|\boldsymbol{u}| \leqslant 1$. For the reservoir simulation, a cross-sectional average of this factor has been verified to be at least 0.98 , an insignificant deviation from unity.

In the second model, following the literature, we assume a linear combination of Coulomb friction and a term expressing the influence of the rate of shear. In these models, the stress tensor is essentially decomposed into a frictional and a collisional part (see the review by Jackson 1986). The frictional part is then often modelled with Coulomb's law. In Bagnold's (1954) seminal work, the closing of the collisional part with a shear stress proportional to the square of the rate of the shear is proposed. In later work, based upon kinetic theory (see the review of Goldhirsh 2003), the square of the rate of strain is often replaced by the product of the square root of granular temperature and rate of strain (see Lun et al. 1984 and Johnson et al. 1990). We find that after division by $\alpha \rho_{p} g_{n} h$ a similar collisional stress contribution leads to a frictional term $\mu$ proportional to $F^{2}$, since both the root of the granular temperature and the rate of strain are proportional to $u$. The inclusion of collisional stresses in rheological theory therefore leads to reduced friction in the contraction since $F$ is relatively low in the contraction for steady flows.

A smooth bottom surface instead of a bottom formed by fixed spheres causes modelling complications. Both Bagnold's arguments and kinetic theory concern the shear between two layers of particles and not the shear or slip between a wall and a layer of particles. For a smooth surface, collisional theory is therefore usually combined with Coulomb friction. For a perfectly flat frictional bottom, as in our 

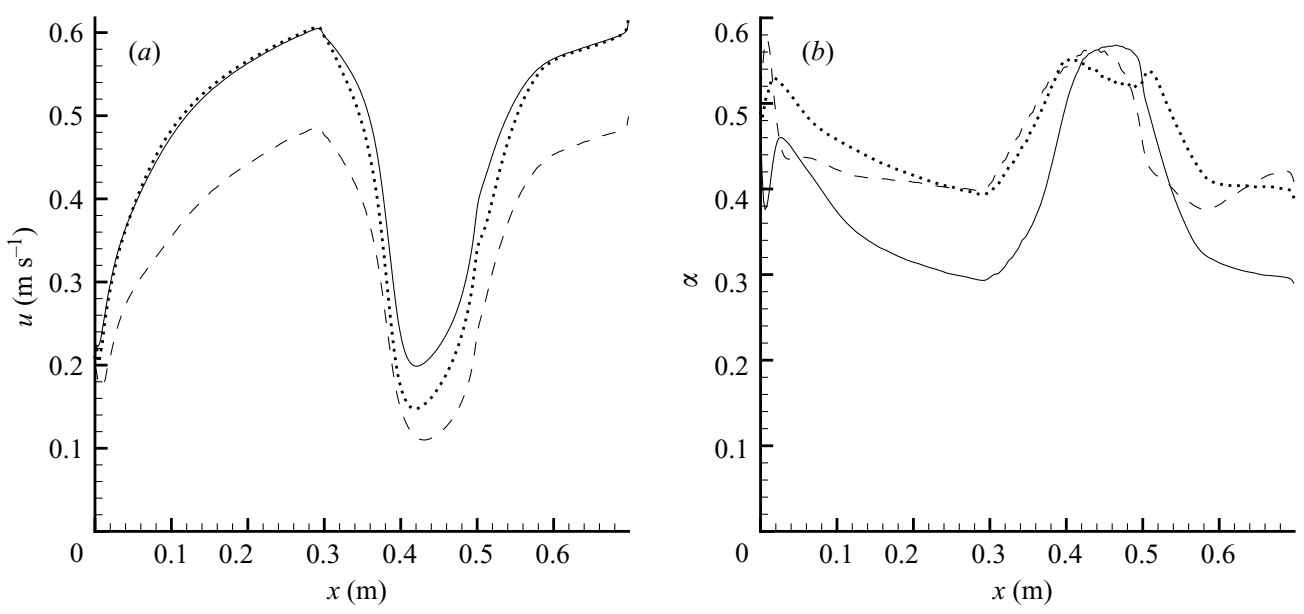

FIGURE 17. Two width-averaged profiles near the wall at $z=0.5 \mathrm{~mm}$ (dashed) and $z=1.5 \mathrm{~mm}$ (dotted) compared with the cross-sectionally averaged steady-state profiles (solid) for $(a)$ the streamwise velocity and $(b)$ the volume fraction.
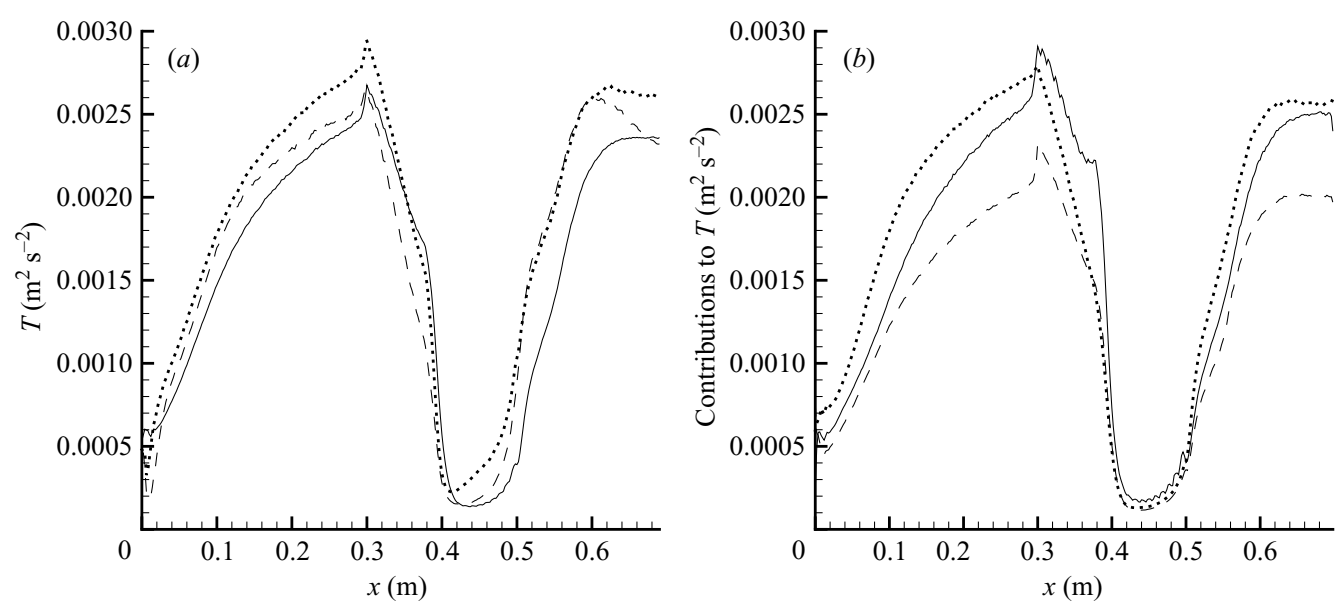

Figure 18. (a) Cross-sectionally averaged $T$ profiles near the bottom wall at $z=0.5 \mathrm{~mm}$ (dashed) and $z=1.5 \mathrm{~mm}$ (dotted) compared with the depth-averaged steady-state profiles for $T$. (b) The three distinct contributions of the depth-averaged $T$, which together sum to $T$ : streamwise (solid), transversal horizontal (dashed) and bottom wall-normal contribution (dotted).

simulations, the velocity profile exhibits shear, see figure 17 . When the bottom friction coefficient is not lower than the internal friction coefficient, the near-wall velocity is naturally reduced because a particle in the bottom layer has higher probability of contact with the bottom plane than particles in the adjacent layers. In addition, figure $17(b)$ shows the volume fraction, which in theoretical works is often assumed to be independent of depth, while the simulation results show a relatively high concentration of particles near the bottom. The profile of granular temperature, however, is almost independent of depth: the wall and depth-averaged temperature in figure 18(a) are almost the same.

Johnson et al. (1990) proposed combining the kinetic collisional model by Lun et al. (1984) with frictional terms and applied the model to chute flows with a flat 
bottom made of aluminium. Although the kinetic collisional theory is based on binary collisions, a concept which has limitations at high volume fractions, Lun et al. (1984) and Johnson et al. (1990) both mention that the constitutive equations formulated by Lun et al. are appropriate for the entire range of volume fractions. We test the applicability of their theory to the contraction flow.

The constitutive model for the collision stress tensor according to Lun et al. (1984) is, after a few simplifications

$$
\boldsymbol{\sigma}_{c} \approx-\left(\rho_{p} \alpha T\left(1+4 \alpha g_{0}\right)-\mu_{b} \nabla \cdot \boldsymbol{u}\right) \boldsymbol{I}+1.2 \mu_{b} \boldsymbol{S},
$$

where $\boldsymbol{I}$ is the unity tensor, $\boldsymbol{S}$ the deviatoric part of the strain and

$$
g_{0}=1 /\left(1-\left(\alpha / \alpha_{\max }\right)^{1 / 3}\right), \quad \mu_{b}=\frac{8}{3} \rho_{p} d \alpha^{2} g_{0} \sqrt{T / \pi},
$$

where $d$ is the particle diameter and $\alpha_{\max }=0.65$. We substituted $\eta=\frac{1}{2}(1+e)=1$ in the original equations given by Lun et al. since in our simulations $\eta=0.985$. From the viscosity proposed by Lun et al., we only retained the part $\mu_{b}$ in the coefficient in front of $\boldsymbol{S}$, which is the main contribution to the viscosity if $\alpha>0.3$. Johnson et al. (1990) adopted this kinetic theory, but added frictional parts to the stress tensor: $N_{f}$ for normal components and $N_{f} \sin \phi_{i}$ for tangential components where $\phi_{i}$ is the internal friction angle. $N_{f}=0.05(\alpha-0.5)^{2} /\left(\alpha_{\max }-\alpha\right)^{5}$ if $\alpha>0.5$ and 0 otherwise. The stress boundary condition at the bottom of the chute, is (Johnson et al. 1990; see also Hui et al. 1984)

$$
S_{w}=\phi^{\prime} \pi \rho_{p} \alpha\left|u_{s l}\right| \sqrt{3 T} /\left(6 g_{0} \alpha_{\max }\right)+N_{f} \tan \phi_{w},
$$

where $\phi_{w}$ is the friction angle between the particles and wall, the sliding velocity $u_{s l}$ equals the velocity of the bottom layer of particles (see figure 17a), and $\phi^{\prime}$ is a specularity coefficient, equal to 0.25 for aluminium.

We evaluate the frictional-kinetic theory for a one- and two-dimensional description of the contraction, which means that we use depth and cross-sectionally averaged profiles to evaluate the constitutive equations, with the exception of $S_{w}$ which is calculated with use of the values of $T$ and $u$ at $z=0$.

The isotropic part of the constitutive stresses in (5.1) can be interpreted as a granular pressure, such that a frictional-kinetic model for the granular pressure becomes

$$
p_{\text {mod }}=N_{f}+\rho_{p} \alpha T\left(1+4 \alpha g_{0}\right)-\mu_{b} \nabla \cdot \boldsymbol{u} .
$$

If we evalute $p_{\text {mod }}$ for depth-averaged quantities we can compare this with the depthaveraged 'static' pressure, $\frac{1}{2} \alpha \rho_{p} g_{n} h$. The comparison is shown in figure 19, where we also plot $\frac{1}{2} p_{1}$, where $p_{1}$ is the pressure at the wall directly computed from the discrete-particle simulation without using the constitutive equation. Two important conclusions can be drawn from this figure. First, the 'static' pressure approximation is a quite accurate approximation of the actual pressure $p_{1}$. The small difference between $p_{1}$ and $\alpha \rho_{p} g_{n} h$ can be explained by the term $u \partial w / \partial x$, which balances the pressure gradient in the wall-normal direction, as expressed by the non-averaged momentum equation in the $z$-direction. The second conclusion is that although the magnitude of $p_{\text {mod }}$ is wrong, its trend is correct as the pressure increases in the contraction. The frictional contribution $N_{f}$ is essential for this increase.

The frictional coefficient $\mu$ in our one-dimensional equations can be calculated from the kinetic theory by cross-sectionally and depth-averaging the divergence of the granular stress tensor, applying Leibniz' rule, assuming zero stress boundary conditions at the free surface, subtracting the hydrostatic pressure term and dividing 


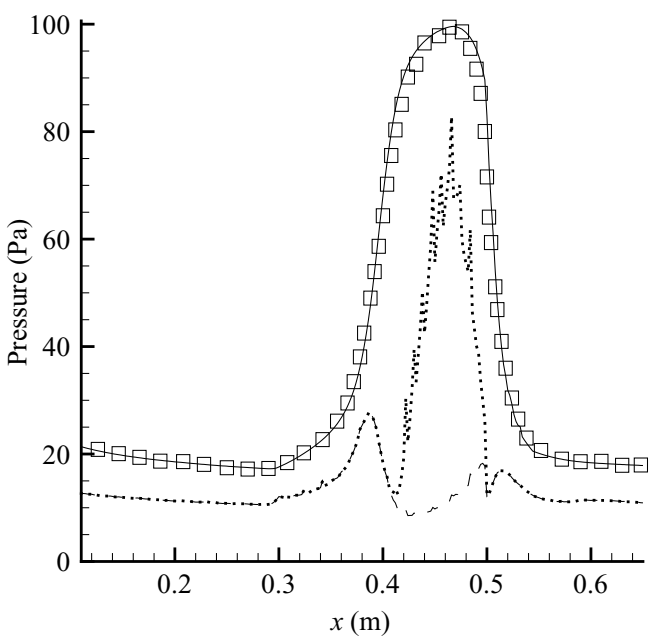

FIGURE 19. Frictional-kinetic pressure $p_{\text {mod }}$ with (dotted) and without $N_{f}$ (dashed), compared with the 'hydrostatic' pressure $\frac{1}{2} \rho_{p} \alpha g_{n} h$ (solid) and the actual pressure $\frac{1}{2} p_{1}$ (squares).

the result by $\rho_{p} \alpha g_{n} h$ :

$$
\begin{aligned}
\mu & =-\frac{\partial h}{\partial x}-\frac{1}{\rho_{p} \alpha g_{n} b h} \int_{-b / 2}^{b / 2} \int_{0}^{h} \nabla \cdot \sigma_{c} \mathrm{~d} z \mathrm{~d} y \\
& \approx-\frac{\partial h}{\partial x}+\frac{1}{\rho_{p} \alpha g_{n} h}\left[\frac{\partial\left(h p_{m o d}\right)}{\partial x}-\frac{\partial}{\partial x}\left(\frac{6}{5} h \mu_{b}\left(\frac{\partial u}{\partial x}-\frac{1}{3} \nabla \cdot \boldsymbol{u}\right)\right)+S_{w}\right] .
\end{aligned}
$$

The terms inside the square brackets represent the depth-averaged $\partial \sigma_{x x} / \partial x$, while $S_{w}$ results from depth-averaging the shear-stress term $\partial \sigma_{x z} / \partial z$. The divergence of the velocity in (5.6) represents

$$
\frac{1}{h} \int_{0}^{h} \nabla \cdot \boldsymbol{u} \mathrm{d} z \approx \frac{\partial u}{\partial x}+\frac{u}{h} \frac{\partial h}{\partial x} .
$$

The last term in (5.7) arises from the integration of $\partial w / \partial z$ and application of the kinematic boundary condition at the free surface.

According to the reservoir simulation data, $\mu$ calculated from (5.6) appears to be an inaccurate approximation of the actual $\mu$ (figure 20). However, the friction is reduced in the reservoir, which means that the model is in principle able to explain the occurrence of reservoirs in contraction flows. The shear-stress boundary contribution $S_{w}$ appears to be the dominant term in (5.6). It is severely reduced inside the contraction, due to the reduction of granular temperature and slip velocity at the bottom of the chute.

To verify whether the poor accuracy of the model is caused by the reduction to one dimension, we also evaluated the model for two-dimensional flow (only depthaveraged). Results were not improved, as shown by the circles in figure 20, which represent the two-dimensional friction coefficient after cross-sectionally averaging. The two-dimensional model is similar to (5.6), but the expression for the divergence is extended with $y$-derivatives, while $y$-derivatives arising from the strain-component $S_{x y}$ also occur. 


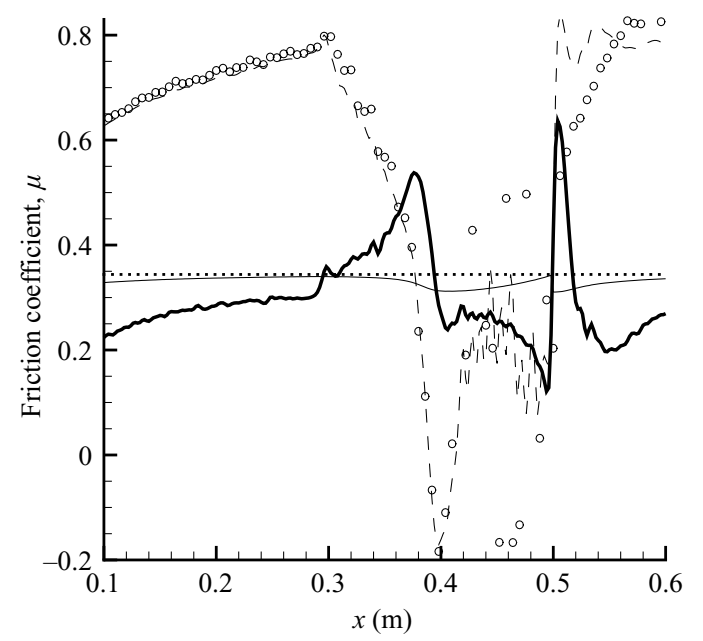

FIGURE 20. Models for $\mu$ compared with the actual $\mu$ (thick solid): constant Coulomb friction (dotted); equation (5.6), one-dimensional version (dashed) and extension to two-dimensional (circles); Savage \& Hutter model (thin solid).

The third model we wish to validate is that of Savage \& Hutter (1991) with

$$
\mu=1.25 \tan \delta_{0}(1-\exp (-c F))(1+0.453 h / b),
$$

where model constant $c$ equals 0.64 and $\delta_{0}$ is a quasi-static value. We put $1.25 \tan \delta_{0}$ equal to 0.344 , the friction coefficient used in the simulation. An interesting feature of this model is that effects of sidewalls are included through the last factor. Since we do not know the values of the other constants in (5.8) for our specific case, we use the values mentioned by Savage $\&$ Hutter. Although the chute in their experiments had some roughness, it was not coated with particles of the same material and size as the flow. Instead PVC, writing paper and sandpaper were used, and in particular the former two were reasonably flat for particle diameters of a few $\mathrm{mm}$. According to expression (5.8), $80 \%$ of the friction is expressed by Coulomb's law, while $20 \%$ is variable and represents the effects of the rate of shear. The latter part is expressed in $F$, and indeed the friction decreases if $F$ decreases. The Savage \& Hutter model will be able to predict a reservoir, since it predicts a significant reduction of friction inside the contraction (figure 20). However, the peak of friction in the first part of the contraction is not covered.

None of the models represented in figure 20 is able to reproduce the strong increase of friction in the first half of the contraction. This strong increase in this part of the flow may well be caused by the strong shock, since the shock is at the same location as the first peak of friction. Apparently, the dissipative character of the shock is not recognized by the constitutive equations of friction that we discuss.

The fourth model (Louge \& Keast 2001) differs from the previous models because friction decreases with $F$ (see equation (54) in Louge \& Keast 2001). It is therefore not able to explain our observations. Nevertheless, using our numerical database, we calculated the friction coefficient prescribed by equation (59) in Louge \& Keast,

$$
\mu=\mu_{E}-f_{L} F^{2},
$$

where $f_{L}$ is a positive function and $\mu_{E}$ is the friction coefficient between particles and the bottom of the chute. The result was very similar to the straight, dotted, Coulomb 
line in figure 20. The difference was less than $1 \%$ and a slight increase of friction in the contraction was observed.

Finally, we mention the model proposed by Pouliquen \& Forterre (2002). This model was not calibrated for flat planes, but for rough inclines with spherical particles fixed to the surfaces. Hence, it is not valid for our experiments. We only remark that in this model friction also increases with $F$, which is a minimum requirement to capture steady reservoirs in a contraction.

\section{Conclusions}

In this paper, we presented a series of granular experiments on supercritical shallow flows through a contraction on an inclined plane. In line with Akers \& Bokhove's (2007) hydraulic experiments in a horizontal flume, we observed three different flow states for granular flows on inclined planes: (I) smooth supercritical flow, (II) flow with a non-steady backward travelling bore or a steady jump upstream of the contraction region, and (III) a steady reservoir with a standing jump in the contraction. Four distinct regimes were observed in the phase plane of contraction width $b_{c}$ and supercritical upstream Froude number $F_{0}>1$, summarized in figure 12 . Three of these regimes corresponded to the flow states (I), (II) and (III), while a fourth regime (IV) represented observations of hysteretic flows. Regime (III) concerns flow states with relatively low supercritical Froude number $\left(1<F_{0}<4\right)$, while regime (IV) corresponded to ones with larger Froude number $\left(F_{0}>4\right)$. In the latter regime, two possible flow states were observed for specific points $\left(b_{c}, F_{0}\right)$. Short temporal disturbances of the flow were sufficient to switch the flow from one state (I) to another (II or III). Significant variations of the porosity were measured, leading to quantitative changes, but qualitative features of the experimental regimes did not change due to these porosity variations.

Theoretical analysis showed that friction is essential, in particular to understand the formation of a steady reservoir. Friction forces in such reservoirs are inferred as being relatively low compared to their upstream values. Classical, inviscid and incompressible hydraulic theory has been extended to include viscous and compressibility effects represented by acceleration integrals. The extended theory with approximated acceleration integrals led to two demarcation lines, dividing the phase plane into four quadrants, denoted by the four regimes in figure 12. Contrastingly, in classical hydraulics these demarcation lines cross at $\left(b_{c} / b_{0}, F_{0}\right)=(1,1)$ leading to only three supercritical regimes. In our granular flow experiment, friction shifts these lines such that they cross in the middle of the phase plane, around $(0.2,4.0)$. A new regime with a steady reservoir inside the contraction emerges as a consequence. Theory and observations show that the flow accelerates in the contraction. Friction is hence reduced in the contraction. Simple models in the literature, analysed in $\S 5$, support this phenomenon because it corresponds to the observed decrease of the macroscopic velocity scale and increase of the macroscopic length scale, in the depth in the present instance. Strikingly, the flow regimes for (water and) spherical glass beads and the lighter, non-spherical poppy seeds do not coincide in the phase diagram. This suggests that the shape and density of the granular material may have to be included to permit a possible collapse of the data in one phase diagram.

Discrete-particle simulations were performed for four different widths, $b_{c}$, and one Froude number, $F_{0}$. The maximum number of particles in the system was about 378000 and the equations were integrated for more than $10 \mathrm{~s}$ (physical time), using a linear spring/dash-pot model for the contact forces. Quantitative agreement was 
observed by comparing depth, porosity, two-dimensional velocity patterns, reservoir length and the demarcation between flow regimes I and III between simulations and experiments. The crucial role of friction in the reservoir formation was confirmed by the simulations from statistics of the contact forces. The simulations also showed that for smooth supercritical flows of glass beads the friction in the contraction is increased instead of reduced, see figure 16. The simulations revealed that the effect of increased porosity and the effect of increased friction in the contraction cancel out to some extent, while the increase of friction for weakly contracted flows was confirmed. Furthermore, the simulations strongly indicated that porosity influences friction in a non-trivial way.

Several friction or constitutive laws reported in the literature have been evaluated using the numerical database. Most constitutive laws confirm the reduction of friction if the Froude number decreases, as we observed in the contraction. The magnitude of the theoretical predictions often did not correspond with our simulations. None of the models was able to reproduce the increase of friction just before the reservoir. It seems that the constitutive equations which we considered have problems in accounting for the dissipation caused by granular bores and jumps. It remains a challenge to model shallow granular flows accurately by continuum approaches.

The authors are grateful to W. Leppink for his technical support and N. G. Deen for his instructions on handling the PIV method. O. B., M. A. and A. W. V. acknowledge support via the Royal Netherlands Academy of Arts and Sciences, and the Institute for Mechanics, Processes and Control-Twente, respectively. O. B. thanks Professor H.-U. Schmincke for his expert guidance during the 2002 field trip in the volcanic Eifel in Germany, and the discussions on the Laacher See eruption. The idea for the experiments was born during this field trip.

\section{Appendix A. Comparison of granular and hydraulic flows}

Two water experiments have been investigated to assess whether the reservoir state would also occur for an incompressible fluid under similar experimental conditions. We will show that such a state exists, which then implies that the steady granular reservoir is not primarily caused by compressibility. Akers (2005) and Akers \& Bokhove (2007) also performed experiments with water through a contraction. In their case, the chute was horizontal and had larger dimensions. They essentially observed the same flow states for water as we reported here for granular flows. They considered the hysteresis phenonemon in detail and showed that classical hydraulic theory (by adaptation of Baines \& Whitehead 2003) applied to one-dimensional equations, after averaging across the chute, provided a leading-order explanation of the flow phenomena observed.

A snapshot of a water reservoir is shown in figure 21(a). The flow seems laminar before the shock and turbulent in the reservoir behind the shock, whereas the granular flow stays laminar except across the shock. In contrast to the V-shaped shock front for reservoirs in granular flows, it is straight in the water experiments (cf. Akers \& Bokhove 2007). The results for experimental sets S9 and S10 (table 1) have been collected in a phase diagram, shown in figure 21(b). The representation of the water experiments in the phase diagram is similar to the representation of the granular experiments around $F_{0} \approx 4$ (compare figure 7).

Depth measurements at different locations and the corresponding values of $F_{c}$ were given in figure 8 . The depth of the water layer was measured with an ordinary ruler 
(a)

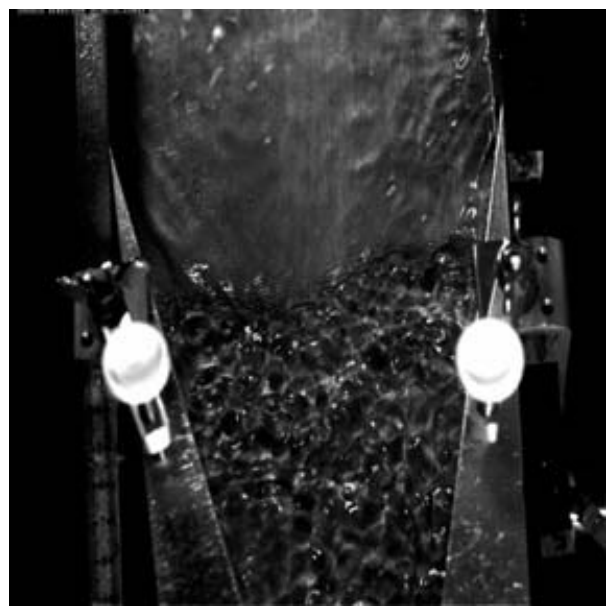

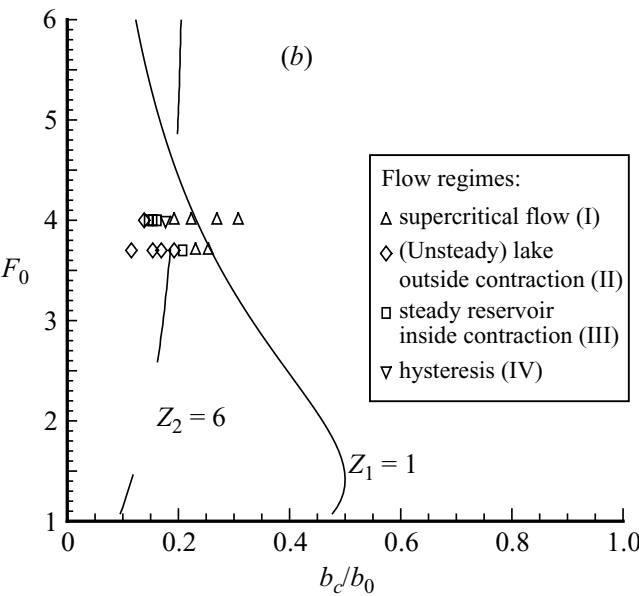

Figure 21. (a) A snapshot of a steady reservoir state for an experiment with water. $(b)$ The experimental results for water collected in a phase diagram together with critical (solid) and shock curves (dashed) found with hydraulic theory including frictional effects.

touching the bottom of the chute (giving measurement errors of about $0.2 \mathrm{~mm}$ ); due to the effect of surface tension the electronic ruler used before was inappropriate.

The water and granular experiments shown in figure 8 correspond to roughly the same $F_{0}$. Surprisingly $h_{0}, u_{0}$ and mass flux $\left(\alpha_{0} \rho_{p} h_{0} u_{0} b_{0}\right.$ for glass beads), and consequently the effective density ( $\alpha_{0} \rho_{p}$ for glass beads), are approximately the same for experiments $\mathrm{S} 1$ and $\mathrm{S} 10$, see table 1. While in the granular lake $\alpha_{c} / \alpha_{0}=1.65$, we observe from figure 8 that the depth of the granular reservoir is about 1.5 times smaller than the depth of the water reservoir. For the water and granular examples, the ratio $\alpha_{c} h_{\text {lake }} /\left(\alpha_{0} h_{0}\right)$ is quite similar. This suggests that the effect of porosity is mainly visible in the depth downstream of the shock, and that there is compaction of the granular layer in the reservoir due to gravity.

We finally show how the value $Z_{2}=6$ can be approximately obtained for water. First, we remark that the dashed line in figure 21 , modelled with an isoline value $Z_{2}=6$, corresponds to a steady reservoir with length $L_{l}=0.20 \mathrm{~m}$. According to figure 21 the flow is turbulent in the reservoir. Thus we know the friction by using the standard surface skin friction coefficient based on the bulk velocity. For turbulent flow, we find $a_{f}=\tan \phi-c_{f} F^{2}$, which shows that the acceleration increases if the local Froude number $F=u / \sqrt{g_{n} h}$ decreases. In the contraction $F<1$ and $c_{f}<0.01$ (Pope 2000) if we just adopt the skin friction coefficient for turbulent channel flow at low Reynolds number. This means that $A_{c}-A_{s} \approx L_{l} a_{f}>0.0084 \mathrm{~m}$, such that $Z_{2}>5$, which is close to $Z_{2}=6$.

\section{Appendix B. Shock relations}

The shock relations arising from (3.1), in the absence of friction and forcing terms, are

$$
\left[\alpha h\left(\boldsymbol{v} \cdot \hat{\boldsymbol{n}}-S_{n}\right)\right]=0 \quad \text { and } \quad\left[\alpha h \boldsymbol{v}\left(\boldsymbol{v} \cdot \hat{\boldsymbol{n}}-S_{n}\right)\right]+\left[\frac{1}{2} g_{n} \alpha h^{2}\right] \hat{\boldsymbol{n}}=0
$$

(Shapiro 1953) with square brackets denoting the jump in a quantity across a shock, $v=\left(0_{1} v\right)$ and $\hat{\boldsymbol{n}}$ the unit vector normal to a shock. In one dimension, the shock 
relations (B 1) reduce to

$$
\begin{aligned}
\left(u_{+}-S_{\alpha}\right) \alpha_{+} h_{+} & =\left(u_{0}-S_{\alpha}\right) \alpha_{0} h_{0}, \\
\left(u_{+}-S_{\alpha}\right) \alpha_{+} h_{+} u_{+}+\frac{1}{2} \alpha_{+} g_{n} h_{+}^{2} & =\left(u_{0}-S_{\alpha}\right) \alpha_{0} h_{0} u_{0}+\frac{1}{2} g_{n} \alpha_{0} h_{0}^{2}
\end{aligned}
$$

with $S_{\alpha}=S_{n}$. Further manipulation yields

$$
\left(u_{+}-S_{\alpha}\right)^{2}=\frac{1}{2} g_{n} \frac{\alpha_{0} h_{0}}{\alpha_{+} h_{+}} \frac{\left(\alpha_{+} h_{+}^{2}-\alpha_{0} h_{0}^{2}\right)}{\left(\alpha_{+} h_{+}-\alpha_{0} h_{0}\right)},
$$

which reduces to (3.23) for $u_{+}=0$. In the steady case, $S_{n}=0$ and (B 1) becomes

$$
[\alpha h \boldsymbol{v} \cdot \hat{\boldsymbol{n}}]=0, \quad\left[\alpha h(\boldsymbol{v} \cdot \hat{\boldsymbol{n}})^{2}\right]+\left[\frac{1}{2} g_{n} \alpha h^{2}\right]=0 \quad \text { and } \quad[\alpha h \boldsymbol{v} \cdot \hat{\boldsymbol{\tau}} \boldsymbol{v} \cdot \hat{\boldsymbol{n}}]=0
$$

with $\hat{\boldsymbol{\tau}}$ the unit vector tangential to the shock. The one-dimensional version of (B 5) is used in (3.14).

Next, we consider steady flow along a wall with a sudden inclination of angle $\theta_{c}$. Assume that an oblique shock arises with an angle $\theta_{s}>\theta_{c}$. The uniform inflow has depth $h_{0}$, speed $u_{0}$, and volume fraction $\alpha_{0}$. The flow behind the shock has speed $u_{+}$ parallel to the wall, depth $h_{+}>h_{0}$ and volume fraction $\alpha_{+}$. Following Shapiro (1953; see Al-Tarazi et al. 2006), we obtain the extended angle-shock relations:

$$
2 F_{0}^{2} \sin ^{2} \theta_{s}=\frac{1}{h_{0}} \frac{\alpha_{+} h_{+}}{\alpha_{0} h_{0}} \frac{\alpha_{0} h_{0}^{2}-\alpha_{+} h_{+}^{2}}{\alpha_{0} h_{0}-\alpha_{+} h_{+}} \quad \text { and } \quad \frac{\alpha_{+}}{\alpha_{0}} \frac{h_{+}}{h_{0}}=\frac{\tan \theta_{s}}{\tan \left(\theta_{s}-\theta_{c}\right)}
$$

with Froude number $F_{0}^{2}=u_{0}^{2} /\left(g_{n} h_{0}\right)$. When $\alpha_{+}=\alpha_{0}$, (B $\left.6 a, b a\right)$ is equivalent to (4.2) in Gray et al. (2003). For constant porosity, (B 6a,b) reduces to (16) in Hakonardottir \& Hogg (2005).

Thus, relations (B $6 a, b)$ imply that given the upstream inflow values summarized in $F_{0}$, the ratio $h_{+} / h_{0}$ and the inclination angle $\theta_{c}$ of the contraction, we can find the shock angle $\theta_{s}$ and the porosity ratio $\alpha_{+} / \alpha_{0}$. Using these expressions, the differences in results between Gray et al. and Hakonardottir \& Hogg (2005) may be explained by porosity effects if we assume that in the former experiment the porosity jump was relatively large and in the latter relatively small. The diameters of particles in these experiments were very different, such that $h_{0} / d$, with $d$ the particle diameter, in the former experiment equalled 4 and in the latter about 44 . In the former case porosity has more influence than in the latter, since for high $h_{0} / d$, volume fraction $\alpha_{0}$ is closer to its maximum value than for low $h_{0} / d$.

\section{REFERENCES}

Akers, B. 2005 Shallow water flow through a contraction. Report Geophysical Fluid Dynamics Fellowship Program, Woods Hole Oceanographic Institution. http://gfd.whoi.edu/proceedings.html

Akers, B. \& Bokhove, O. 2007 Hydraulic flow through a contraction: multiple steady states. http: eprints.eemcs.utwente.nl

Al-Tarazi, M., Bokhove O., Kuipers, J. A. M., van Sint Annaland, M. \& Vreman, A. W. 2006 Reservoir formation in shallow granular flows through a contraction. Mathematical Communications, Department of Applied Mathematics. ISSN 0169-2690, http://eprints.eemcs.utwente.nl

Augenstein, D. A. \& Hogg, R. 1978 An experimental sTUdy of the flow of dry powders over inclined surfaces. Powder Technol. 19, 205-215.

BAGNOLD, R. A. 1954 Experiments on a gravity free dispersion of large solid spheres in a Newtonian fluid under shear. Proc. R. Soc. Lond. A 225, 49-63.

Baines, P. G. \& Whitehead, J. A. 2003 On multiple states in single-layer flows. Phys. Fluids 15, 398-407. 
Brennen, C. E., Sieck, K. \& Palaski, J. 1983 Hydraulic jumps in granular material flow. Powder Technol. 35, 31-37.

Campbell, C. S. \& Brennen, C. E. 1985 Computer simulations of granular shear flows. J. Fluid Mech. 151, 167-188.

Campbell, C. S., Brennen, C. E. \& Sabersky, R. H. 1985 Flow regimes in inclined open-channel flows of granular materials. Powder Technol. 41, 77-82.

Cundall, P. A. \& Strack, O. D. L. 1979 A discrete numerical model for granular assemblies. Geótechnique 29, 47.

GDR MiDi 2004 On dense granular flows. Eur. Phys. J. E 14, 341-365.

Goldhirsch, I. 2003 Rapid granular flows. Annu. Rev. Fluid Mech. 35, 267-293.

Goldschmidt, M. J. V., Beetstra, R. \& Kuipers, J. A. M. 2004 Hydrodynamic modelling of dense gas-fluidised beds: comparison and validation of three-dimensional discrete particle and continuum models. Powder Technol. 142, 23-47.

Gray, J. M. N. T., TaI, Y.-C. \& Noelle, S. 2003 Shock waves, dead zones and particle-free regions in rapid granular free-surface flows. J. Fluid Mech. 491, 161-181.

HafF, P. K. 1983 Granular flow as a fluid mechanical phenomenon. J. Fluid Mech. 134, 401-430.

HakonardottiR, K. M. \& HogG, A. J. 2005 Oblique shocks in rapid granular flows. Phys. Fluids 17, 077101.

van der Hoef, M. A., Ye, M., van Sint Annaland, M., Andrews IV, A. T., Sundaresan, S. \& Kuipers, J. A. M. 2006 Multi-scale modelling of gas-fluidized beds. Adv. Chem. Engng 31, 65.

Hui, K., Haff, P. K., Ungar, J. R. \& JACKSON, R. C. 1984 Boundary conditions for high-shear grain flows. J. Fluid Mech. 145, 223-233.

JACKSON, R. 1986 Some features of the flow of granular materials and aerated granular materials. J. Rheology 30, 907-930.

Johnson, P. C., NotT, P. \& JACKsOn, R. C. 1990 Frictional-collisional equations of motion for particulate flows and their application to chutes. J. Fluid Mech. 210, 501-535.

Lun, C. K. K., Savage, S. B., Jefrrey, D. J. \& Chepurniy, N. 1984 Kinetic theories for granular flow: inelastic particles in Couette flow and slightly inelastic particles in a general flow field. J. Fluid Mech. 140, 223-256.

Louge, M. Y. \& Keast, S. C. 2001 On dense granular flows down flat frictional inclines. Phys. Fluids 13, 1213-1233.

Pope, S. B. 2000 Turbulent Flows. Cambridge University Press.

Pouliquen, O. 1999 Scaling laws in granular flows down a rough inclined plane'. Physics of fluids 11, 542-548.

Pouliquen, O. \& Forterre, Y. 2002 Friction law for dense granular flows: application to the motion of a mass down a rough inclined plane. J. Fluid Mech. 453, 133-151.

Savage, S. B. \& Hutter, K. 1989 The motion of a finite mass of granular material down a rough incline. J. Fluid Mech. 199, 177-215.

SAvage, S. B. \& Hutter, K. 1991 The dynamics of avalanches of granular materials from initiation to run-out. Part I: Analysis. Acta Mechanica 86, 201-223.

SChmincKe, H.-U. 2000 Vulkanismus. Wissenschaftliche Buchgesellschaft Darmstadt.

Silbert, L. E., Landry, J. W. \& Crest, G. S. 2003 Granular flow down a rough inclined plane: transition between thin and thick piles. Phys. Fluids 15, 1-10.

Shapiro, A. H. 1953 The Dynamics and Thermodynamics of Compressible Fluid Flow. New York: Ronald Press. 